\title{
Proof of the formula of Cachazo, He and Yuan for Yang-Mills tree amplitudes in arbitrary dimension
}

\author{
Louise Dolan ${ }^{a}$ and Peter Goddard ${ }^{b}$ \\ ${ }^{a}$ Department of Physics, University of North Carolina, \\ Cameron Avenue, Chapel Hill, NC 27599, U.S.A. \\ ${ }^{b}$ School of Natural Sciences, Institute for Advanced Study, \\ Einstein Drive, Princeton, NJ 08540, U.S.A. \\ E-mail: ldolan@physics.unc.edu, pgoddard@ias.edu
}

Abstract: A proof is given of the formula, recently proposed by Cachazo, He and Yuan (CHY) for gluon tree amplitudes in pure Yang-Mills theory in arbitrary dimension. The approach is to first establish the corresponding result for massless $\phi^{3}$ theory using the $\mathrm{BCFW}$ recurrence relation and then to extend this to the gauge theory case. Additionally, it is shown that the scattering equations introduced by CHY can be generalized to massive particles, enabling the description of tree amplitudes for massive $\phi^{3}$ theory.

Keywords: Scattering Amplitudes, Duality in Gauge Field Theories, Global Symmetries, String Duality

ARXIV EPRINT: 1311.5200 


\section{Contents}

1 Introduction 1

2 Properties of the CHY scattering equations 3

3 Massless $\phi^{3}$ theory $\quad 6$

4 Inductive proof for (partly) off-shell massless $\phi^{3}$ theory $\quad 8$

$5 \quad$ BCFW proof for massless $\phi^{3} \quad 12$

$\begin{array}{lll}6 & \text { Proof for pure gauge theory } & 15\end{array}$

$\begin{array}{lr}\text { A Properties of Pfaffians } & 19\end{array}$

B Massive $\phi^{3}$ theory $\quad 22$

\section{Introduction}

Recently Cachazo, He and Yuan (CHY) have proposed a compact and relatively simple formula for the sum of all $N$-point tree diagrams for pure Yang-Mills theory in an arbitrary dimension of space-time and a similar formula for the sum of all $N$-point tree diagrams for gravity [1]. These formulae express the $N$-point amplitudes as sums over the solutions for $z_{a}$ to the equations

$$
\hat{f}_{a}(z, k)=0, \quad a \in A, \quad \text { where } \quad \hat{f}_{a}(z, k)=\sum_{\substack{b \in A \\ b \neq a}} \frac{k_{a} \cdot k_{b}}{z_{a}-z_{b}},
$$

and where $k_{a}$ are the momenta of the particles labeled by $a \in A$, with $N=|A|$, which CHY call the scattering equations. It follows from momentum conservation, $\sum_{a \in A} k_{a}=0$, and the condition that the particles are massless, $k_{a}^{2}=0, a \in A$, that this system of equations is invariant under Möbius transformations,

$$
z_{a} \mapsto \zeta_{a}=\frac{\alpha z_{a}+\beta}{\gamma z_{a}+\delta}, \quad a \in A,
$$

in that, if $z=\left(z_{a}\right)$ is a solution to $(1.1), \zeta=\left(\zeta_{a}\right)$ also provides a solution: $\hat{f}_{a}(\zeta, k)=0, a \in A$.

Because of this symmetry under the three-dimensional Möbius group, only $N-3$ of the equations (1.1) are independent, and we can restrict to their solutions by means of the delta functions

$$
\prod_{a \in A}^{\prime} \delta\left(\hat{f}_{a}(z, k)\right) \equiv\left(z_{i}-z_{j}\right)\left(z_{j}-z_{k}\right)\left(z_{k}-z_{i}\right) \prod_{\substack{a \in A \\ a \neq i, j, k}} \delta\left(\hat{f}_{a}(z, k)\right)
$$


which is independent of the choice of $i, j, k \in A$. Under the Möbius transformation (1.2),

$$
\prod_{a \in A}^{\prime} \delta\left(\hat{f}_{a}(z, k)\right) \mapsto \prod_{a \in A}^{\prime} \delta\left(\hat{f}_{a}(\zeta, k)\right)=\prod_{a \in A} \frac{\alpha \delta-\beta \gamma}{\left(\gamma z_{a}+\delta\right)^{2}} \prod_{a \in A}^{\prime} \delta\left(\hat{f}_{a}(z, k)\right),
$$

so that the integrand of

$$
\mathcal{A}_{N}=\int \widehat{\Psi}_{N}(z ; k ; \epsilon) \prod_{a \in A}^{\prime} \delta\left(\hat{f}_{a}(z, k)\right) \prod_{a \in A} \frac{d z_{a}}{\left(z_{a}-z_{a+1}\right)^{2}} / d \omega
$$

is Möbius invariant provided that the function $\widehat{\Psi}_{N}(z ; k ; \epsilon)$ is itself Möbius invariant. In (1.5), we have chosen a cyclic ordering on $A$, with $a+1$ indicating the label next after $a$ in the ordering, say $(1,2, \ldots, N)$ with $N+1 \equiv 1, \widehat{\Psi}_{N}(z ; k ; \epsilon)$ may depend on the momenta $k_{a}$ and polarizations, $\epsilon_{a}$, if any, of the particles, and $d \omega$ denotes the invariant measure on the Möbius group,

$$
d \omega=\frac{d z_{r} d z_{s} d z_{t}}{\left(z_{r}-z_{s}\right)\left(z_{s}-z_{t}\right)\left(z_{t}-z_{r}\right)} .
$$

In [1], CHY propose, and present evidence for, an explicit form for $\widehat{\Psi}_{N}(z ; k ; \epsilon)$ for the sum of $\mathrm{N}$-gluon tree diagrams in pure gauge theory, in an arbitrary dimension of space-time, for a given order of the external gluons (to be multiplied by an appropriate color factor). In order to give a precise interpretation of (1.5) that includes a sum over all, possibly complex, solutions of the equations (1.1), CHY rewrite the expression as a contour integral

$$
\mathcal{A}_{N}=\oint_{\mathcal{O}} \widehat{\Psi}_{N}(z ; k ; \epsilon) \prod_{a \in A}^{\prime} \frac{1}{\hat{f}_{a}(z, k)} \prod_{a \in A} \frac{d z_{a}}{\left(z_{a}-z_{a+1}\right)^{2}} / d \omega
$$

where the contour $\mathcal{O}$ encloses all the solutions of (1.1) and $\prod^{\prime}$ is defined as in (1.3).

Here, following the approach we used in [2], to establish the complete equivalence of gluon tree amplitudes in open twistor string theory [3] to the corresponding amplitudes in $N=4$ super Yang-Mills theory, we prove these CHY formulae using the BCFW relations [4], by first showing that taking $\widehat{\Psi}_{N}(z ; k ; \epsilon)$ constant in (1.5) gives the tree amplitudes for massless $\phi^{3}$ theory. [In [5], CHY have also noted that taking $\widehat{\Psi}_{N}(z ; k ; \epsilon)$ constant in (1.5) can provide the amplitudes for a scalar theory, but they consider a theory with $\mathrm{U}(N) \times \mathrm{U}(\tilde{N})$ symmetry.]

The CHY equations $\hat{f}_{a}(z, k)=0, a \in A$, can be modified to describe massive particles (although we only need the massless case as a precursor to our proof of the CHY proposal for gauge theory tree amplitudes). Having chosen a cyclic ordering on $A$, we consider

$$
\check{f}_{a}(z, k)=\sum_{\substack{b \in A \\ b \neq a}} \frac{k_{a} \cdot k_{b}}{z_{a}-z_{b}}+\frac{m^{2}}{2\left(z_{a}-z_{a+1}\right)}+\frac{m^{2}}{2\left(z_{a}-z_{a-1}\right)}, \quad a \in A,
$$

where, in the signature we are using, $k_{a}^{2}=m^{2}, a \in A$. This system of equations is again Möbius invariant, and now (1.7) produces the tree amplitudes for a $\phi^{3}$ theory for a scalar 
particle of mass $m$. However, unlike the original, these modified CHY equations depend on the order of particles which previously only entered through what one might regard as the measure factor in the integrand of (1.5) or (1.7).

After reviewing the general properties of the CHY scattering equations in section 2, we give a calculation in this formalism of the elementary cases of the 4- and 5-point amplitudes in massless $\phi^{3}$ in section 3 , noting that if we have chosen $z_{1} \rightarrow \infty$, the formulae would apply even when $k_{1}$ is off-shell. In section 4 , we give a direct inductive proof of the formula for $\phi^{3}$ theory, using a form of the result that works when one of the momenta is off shell. This approach would be very cumbersome for gauge theory, so we give a second proof in section 5 using the BCFW relations. This analysis is extended in section 6 to give a proof of the formula of CHY [1] for the tree amplitudes of pure gauge theory. Results on Pfaffians, necessary for the discussion of section 6 are described in appendix A. The extension of the results on $\phi^{3}$ theory to the massive case is discussed in appendix B.

\section{Properties of the $\mathrm{CHY}$ scattering equations}

We review some properties of the CHY scattering equations (1.1).

Möbius invariance. This system of equations is Möbius invariant provided that momentum is conserved, i.e. $\sum_{a \in A} k_{a}=0$, and all the particles are massless, i.e. $k_{a}^{2}=0, a \in A$. To see this note that

$$
\sum_{\substack{b \in A \\ b \neq a}} \frac{k_{a} \cdot k_{b} z_{b}}{z_{a}-z_{b}}=-\sum_{\substack{b \in A \\ b \neq a}} k_{a} \cdot k_{b}+z_{a} \sum_{\substack{b \in A \\ b \neq a}} \frac{k_{a} \cdot k_{b}}{z_{a}-z_{b}}=k_{a}^{2}, \quad a \in A,
$$

so that,

$$
\text { if } \zeta_{a}=\frac{\alpha z_{a}+\beta}{\gamma z_{a}+\delta}, \quad \sum_{\substack{b \in A \\ b \neq a}} \frac{k_{a} \cdot k_{b}}{\zeta_{a}-\zeta_{b}}=\frac{\gamma z_{a}+\delta}{\alpha \delta-\beta \gamma} \sum_{\substack{b \in A \\ b \neq a}} \frac{k_{a} \cdot k_{b}\left(\gamma z_{b}+\delta\right)}{z_{a}-z_{b}}=0,
$$

provided that $k_{a}^{2}=0$ for all $a \in A$. Thus, then the system of $N$ equations (1.1) determines the $N$ variables $z_{a}$ only up to Möbius transformation, implying only $N-3$ of them are independent and there must be three relations between them. The equations determine the $N-3$ independent cross-ratios of the $z_{a}$; we are free to specify any three of the $z_{a}$ as we wish and the others are then determined by (1.1).

For general values of $z$, the functions $\hat{f}_{a}(z, k)$ satisfy the relations:

$$
\sum_{a \in A} \hat{f}_{a}(z, k)=\sum_{\substack{a, b \in A \\ b \neq a}} \frac{k_{a} \cdot k_{b}}{z_{a}-z_{b}}=0
$$

independently of the momentum conservation and zero-mass conditions on the $k_{a}$;

$$
\sum_{a \in A} z_{a} \hat{f}_{a}(z, k)=\sum_{\substack{a, b \in A \\ b \neq a}} \frac{z_{a} k_{a} \cdot k_{b}}{z_{a}-z_{b}}=\frac{1}{2} \sum_{\substack{a, b \in A \\ b \neq a}} k_{a} \cdot k_{b}=\frac{1}{2}\left[\sum_{a \in A} k_{a}\right]^{2}=0,
$$


using first the zero-mass conditions, $k_{a}^{2}=0$, and then momentum conservation; and

$$
\sum_{a \in A} z_{a}^{2} \hat{f}_{a}(z, k)=\frac{1}{2} \sum_{\substack{a, b \in A \\ b \neq a}} \frac{\left(z_{a}^{2}-z_{b}^{2}\right) k_{a} \cdot k_{b}}{z_{a}-z_{b}}=\frac{1}{2} \sum_{\substack{a, b \in A \\ b \neq a}}\left(z_{a}+z_{b}\right) k_{a} \cdot k_{b}=-\sum_{a \in A} z_{a} k_{a}^{2}=0 .
$$

From (2.4) and (2.5), we have that

$$
\left|\frac{\partial\left(\hat{f}_{a}, \hat{f}_{b}, \hat{f}_{c}\right)}{\partial\left(\hat{f}_{i}, \hat{f}_{j}, \hat{f}_{k}\right)}\right|=\left|\begin{array}{ccc}
1 & 1 & 1 \\
z_{i} & z_{j} & z_{k} \\
z_{i}^{2} & z_{j}^{2} & z_{k}^{2}
\end{array}\right| \times\left|\begin{array}{ccc}
1 & 1 & 1 \\
z_{a} & z_{b} & z_{c} \\
z_{a}^{2} & z_{b}^{2} & z_{c}^{2}
\end{array}\right|^{-1}=\frac{\left(z_{i}-z_{j}\right)\left(z_{j}-z_{k}\right)\left(z_{k}-z_{i}\right)}{\left(z_{a}-z_{b}\right)\left(z_{b}-z_{c}\right)\left(z_{c}-z_{a}\right)}
$$

(1.3) when $\hat{f}_{d}(z, k)=0$ for $d \neq a, b, c, i, j, k$, which establishes that (1.3) is indeed independent of the choice of $i, j, k \in A$.

Relation to tree amplitude integrands in string theory. The $N$-point tree amplitude in the original bosonic open string theory is given, in Koba-Nielsen variables, by an integral

$$
A_{N}^{\text {string }}=\int V(z ; k) \prod_{a \in A} \frac{d z_{a}}{z_{a}-z_{a+1}} / d \omega
$$

with

$$
V(z, k)=\prod_{1 \leq a<b \leq N}\left(z_{a}-z_{b}\right)^{-\alpha^{\prime} k_{a} \cdot k_{b}} \prod_{a=1}^{N}\left(z_{a}-z_{a+1}\right)^{\alpha_{0}} \equiv U(z, k)^{\alpha^{\prime}},
$$

where the leading trajectory, $\alpha(s)=\alpha_{0}+\frac{1}{2} \alpha^{\prime} s$, with intercept $\alpha_{0}$ and slope parameter $\alpha^{\prime}$, and the particles satisfy the mass condition $k_{a}^{2}=m^{2}$, where $m^{2}=-2 \alpha_{0} / \alpha^{\prime}=-2 a_{0}$. [Scherk obtained $\phi^{3}$ theory by taking $\alpha^{\prime} \rightarrow 0$, at fixed $a_{0}$, in (2.7) in his original paper on the zero-slope (infinite-tension) limit [6]. In this connection see the recent papers by Mason and Skinner [8] and by Berkovits [9].]

Crucially, $V(z, k)$, or equivalently,

$$
U(z, k)=\prod_{1 \leq a<b \leq N}\left(z_{a}-z_{b}\right)^{-k_{a} \cdot k_{b}} \prod_{a=1}^{N}\left(z_{a}-z_{a+1}\right)^{a_{0}}
$$

is Möbius invariant, that is, if $\zeta_{a}$ is given by $(2.2), U(\zeta, k)=V(z, k)$. Then, if we define $\breve{f}_{a}(z, k)$ by

$$
\frac{\partial U(z, k)}{\partial z_{a}}=-\breve{f}_{a}(z, k) U(z, k)
$$

then $\breve{f}_{a}(z, k)$ is given by (1.8) and the Möbius invariance of $U(z, k)$ implies

$$
\breve{f}_{a}(\zeta, k)=\frac{\partial z_{a}}{\partial \zeta_{a}} \breve{f}_{a}(z, k)=\frac{\left(\gamma z_{a}+\delta\right)^{2}}{(\alpha \delta-\beta \gamma)} \breve{f}_{a}(z, k)
$$

so that the set of equations $\breve{f}_{a}(z, k)=0, a \in A$, is Möbius invariant. $\breve{f}_{a}(z, k)=\hat{f}_{a}(z, k)$ when $a_{0}=0$. 
The Möbius invariance of $U(z, k)$ immediately implies the relations (2.3)-(2.5), because, for an infinitesimal Möbius transformation $\delta z_{a}=\epsilon_{1}+\epsilon_{2} z_{a}+\epsilon_{3} z_{a}^{2}$,

$$
\delta U=\sum_{a \in A} \delta z_{a} \frac{\partial U(z, k)}{\partial z_{a}}=0, \quad \text { implying } \quad \sum_{a \in A} \delta z_{a} \breve{f}_{a}=0
$$

that is,

$$
\sum_{a \in A} \breve{f}_{a}(z, k)=0 ; \quad \sum_{a \in A} z_{a} \breve{f}_{a}(z, k)=0 ; \quad \sum_{a \in A} z_{a}^{2} \breve{f}_{a}(z, k)=0 .
$$

Factorization. Consider the situation in which, as the $k_{a}, a \in A$, vary in some specific way maintaining the zero-mass conditions $k_{a}^{2}=0$, two or more of the $z_{a}$ to tend to the same value, $z_{S}$, say. Specifically, suppose $z_{a}=z_{S}+\epsilon x_{a}+\mathcal{O}\left(\epsilon^{2}\right)$, as $\epsilon \rightarrow 0$, for $a \in S$, where $S \subset A$, and $z_{a} \not \neg z_{S}$ for $a \notin S$. Then

$$
\begin{array}{ll}
\hat{f}_{a}(z, k)=\frac{1}{\epsilon} \hat{g}_{a}(x, k)[1+\mathcal{O}(\epsilon)], \quad \hat{g}_{a}(x, k)=\sum_{\substack{b \in S \\
b \neq a}} \frac{k_{a} \cdot k_{b}}{x_{a}-x_{b}}, & a \in S, \\
\hat{f}_{a}(z, k)=\sum_{b \in S} \frac{k_{a} \cdot k_{b}}{z_{a}-z_{S}}+\sum_{\substack{b \notin S \\
b \neq a}} \frac{k_{a} \cdot k_{b}}{z_{a}-z_{b}}+\mathcal{O}(\epsilon), & a \notin S .
\end{array}
$$

Then, as in (2.3),

$$
\sum_{a \in S} \hat{g}_{a}(x, k)=0
$$

implying that if $\hat{g}_{a}=0$ for $a \in S, a \neq s$ (e.g. as the limit of conditions $\hat{f}_{a}=0$ for $a \in S, a \neq s$, as $\epsilon \rightarrow 0$ ), where $s$ is some particular element of $S$, then it follows that $\hat{g}_{s}(x)=0$ as well. Further, multiplying (2.14) by $x_{a}-x_{r}$, for a choice of $r \in S$, and summing over $a \in S$, as in (2.4), gives

$$
\sum_{a \in S}\left(x_{a}-x_{r}\right) \hat{g}_{a}(x, k)=\sum_{\substack{a, b \in S \\ b \neq a}} \frac{x_{a}-x_{r}}{x_{a}-x_{b}} k_{a} \cdot k_{b}=\frac{1}{2} \sum_{\substack{a, b \in S \\ b \neq a}} k_{a} \cdot k_{b}=\frac{1}{2} k_{S}^{2}, \quad k_{S}=\sum_{b \in S} k_{b} .
$$

Then, if $\hat{g}_{a}(x, k)=0, a \in S, a \neq r, s$, we have that

$$
\left(x_{s}-x_{r}\right) \hat{g}_{s}(x, k)=\frac{1}{2} k_{S}^{2}=\left(x_{r}-x_{s}\right) \hat{g}_{r}(x, k),
$$

and, if additionally $g_{r}(x, k)=0$, then $k_{S}^{2}=0$.

Imposing the equations $\hat{f}_{a}(z, k)=0$, for all $a \in A$, and taking the limit $\epsilon \rightarrow 0$,

$$
\sum_{\substack{b \in S \\ b \neq a}} \frac{k_{a} \cdot k_{b}}{x_{a}-x_{b}}=0, \quad a \in S ; \quad \frac{k_{a} \cdot k_{S}}{z_{a}-z_{S}}+\sum_{\substack{b \notin S \\ b \neq a}} \frac{k_{a} \cdot k_{b}}{z_{a}-z_{b}}=0, \quad a \notin S ; \quad k_{S}^{2}=0 .
$$

So, in this limit, the CHY equations (1.1) have in effect factored into two sets: the first set of equations are the CHY equations for the momenta $\left(k_{a}, a \in S ;-k_{S}\right)$, with associated variables $\left(x_{a}, a \in S ; \infty\right)$; and the second set are the equations for the momenta $\left(k_{S} ; k_{a}, a \notin S\right)$, with associated variables $\left(z_{S} ; z_{a}, a \notin S\right)$. 
Specialization. In what follows, in general it will be convenient to take $(r, s, t)$ in (1.6) to be the same as $(i, j, k)$ in (1.3) and further to take $i=1, j=2$ and $k=N$. Then (1.7) becomes

$$
\left(z_{2}-z_{N}\right)^{2} \int_{\mathcal{O}} \widehat{\Psi}_{N}(z ; k ; \epsilon) \prod_{a=3}^{N-1} \frac{d z_{a}}{\hat{f}_{a}(z, k)} \prod_{a=2}^{N-1} \frac{1}{\left(z_{a}-z_{a+1}\right)^{2}},
$$

and making the particular choice $z_{1}=\infty, z_{2}=1, z_{N}=0$, this becomes

$$
\mathcal{A}_{N}=\oint_{\mathcal{O}} \widehat{\Psi}_{N}^{o}(z ; k ; \epsilon) \prod_{a=3}^{N-1} \frac{d z_{a}}{\hat{f}_{a}^{o}(z, k)} \prod_{a=2}^{N-1} \frac{1}{\left(z_{a}-z_{a+1}\right)^{2}}
$$

where $\widehat{\Psi}_{N}^{o}(z ; k ; \epsilon)$ denotes the specialization of $\widehat{\Psi}_{N}(z ; k ; \epsilon)$ to these values and

$$
\hat{f}_{a}^{o}(z, k)=\frac{k_{a} \cdot k_{2}}{z_{a}-1}+\sum_{\substack{b=3 \\ b \neq a}}^{N-1} \frac{k_{a} \cdot k_{b}}{z_{a}-z_{b}}+\frac{k_{a} \cdot k_{N}}{z_{a}}, \quad 3 \leq a \leq N-1 .
$$

To be clear about the position of singularities, it is convenient to rewrite this by setting

$$
\begin{aligned}
f_{a}(z) & =\hat{f}_{a}^{o}(z, k) \prod_{\substack{c=2 \\
c \neq a}}^{N}\left(z_{a}-z_{c}\right)=\sum_{\substack{b=2 \\
b \neq a}}^{N} k_{a} \cdot k_{b} \prod_{\substack{c=2 \\
c \neq a, b}}^{N}\left(z_{a}-z_{c}\right), \\
\mathcal{A}_{N} & =-(-1)^{\frac{1}{2} N(N-1)} \oint_{\mathcal{O}} \frac{\widehat{\Psi}_{N}^{o}(z ; k ; \epsilon)}{\left(1-z_{3}\right) z_{N-1}} \prod_{a=3}^{N-2} z_{a} \prod_{a=4}^{N-1}\left(1-z_{a}\right) \prod_{b=5}^{N-1} \prod_{a=3}^{b-2}\left(z_{a}-z_{b}\right)^{2} \prod_{a=3}^{N-1} \frac{d z_{a}}{f_{a}} .
\end{aligned}
$$

\section{Massless $\phi^{3}$ theory}

In this section we discuss (1.7) in the simplest case, in which $\widehat{\Psi}_{N}(z ; k ; \epsilon)$ is constant, showing how, for $N=4,5,(2.24)$ produces the amplitudes of massless $\phi^{3}$ theory in a form in which the mass-shell condition $k_{1}^{2}=0$ can be relaxed. In the next section, we will give an inductive proof of this partly off-shell formula for general $N$. Specifically, taking $\widehat{\Psi}_{N}(z ; k ; \epsilon)=(-2)^{3-N}$ in $(1.7)$ and $(2.24)$ :

$$
\begin{aligned}
\mathcal{A}_{N}^{\phi} & =\frac{1}{(-2)^{N-3}} \oint_{\mathcal{O}} \prod_{a \in A}^{\prime} \frac{1}{\hat{f}_{a}(z, k)} \prod_{a \in A} \frac{d z_{a}}{\left(z_{a}-z_{a+1}\right)^{2}} / d \omega \\
& =\frac{\varepsilon_{N}}{2^{N-3}} \oint_{\mathcal{O}} \frac{1}{\left(1-z_{3}\right) z_{N-1}} \prod_{a=3}^{N-2} z_{a} \prod_{a=4}^{N-1}\left(1-z_{a}\right) \prod_{b=5}^{N-1} \prod_{a=3}^{b-2}\left(z_{a}-z_{b}\right)^{2} \prod_{a=3}^{N-1} \frac{d z_{a}}{f_{a}},
\end{aligned}
$$

where $\varepsilon_{N}=(-1)^{\frac{1}{2} N(N+1)}, f_{a}$ is given by (2.23), the contour $\mathcal{O}$ encircles the simultaneous solutions of $f_{a}(z, k)=0,3 \leq a \leq N-1$ (but excludes $z_{3}=1$ and $z_{N-1}=0$ ), and we need to sum $\mathcal{A}_{N}^{\phi}$ over the different cyclic orderings of the external legs.

For $N=4$, writing $z=z_{3}$, this gives

$$
\begin{aligned}
\mathcal{A}_{4}^{\phi} & =\frac{1}{2} \oint_{\mathcal{O}} \frac{d z}{(1-z) z\left[k_{3} \cdot k_{2} z+k_{3} \cdot k_{4}(z-1)\right]} \\
& =\frac{k_{3} \cdot\left(k_{2}+k_{4}\right)}{2 k_{3} \cdot k_{2} k_{3} \cdot k_{4}}=\frac{1}{2 k_{3} \cdot k_{4}}+\frac{1}{2 k_{3} \cdot k_{2}}=\frac{1}{s}+\frac{1}{t},
\end{aligned}
$$


where $s=\left(k_{3}+k_{4}\right)^{2}, t=\left(k_{2}+k_{3}\right)^{2}, u=\left(k_{2}+k_{4}\right)^{2}$. Summing over the 6 different cyclic orderings (by permuting $2,3,4$ ), and multiplying by $g^{2} / 4$, where $g$ is the coupling constant, gives the total amplitude $\mathcal{A}_{4}^{\phi \text { tot }}=g^{2} / s+g^{2} / t+g^{2} / u$ (because each term occurs four times), the appropriate result for $\phi^{3}$ theory, $\mathcal{L}=\frac{1}{2} \partial^{\mu} \phi \partial_{\mu} \phi+(g / 3 !) \phi^{3}$.

For the $N$-point function, $\mathcal{A}_{N}^{\phi \text { tot }}$, we need to multiply (3.2) by $(g / 2)^{N-2}$ as we sum over the possible different orderings keeping 1 fixed, because, as we shall prove, $\mathcal{A}_{N}^{\phi}$ gives the sum of the tree diagrams in $\phi^{3}$ theory that can be drawn in planar form for a given ordering of the external momenta [as $1 / s$ in $(3.3)$ can be for the ordering $(1,2,3,4)$ but not the ordering $(1,3,2,4)$ ]. Each tree diagram will contribute to $2^{N-2}$ orderings, treating orderings related by cyclic rotations as equivalent. (If we treat orderings related by anticyclic or cyclic rotations as equivalent, we sum over half as many orderings and each diagram occurs $2^{N-3}$ times, so, in that case, we multiply by $g^{N-2} / 2^{N-3}$.) This is the same counting as in Scherk's original discussion of the zero-slope limit of what became string theory [6]. It would be good to be able to derive (3.2) directly from (1.7) by taking $\alpha^{\prime} \rightarrow 0$. (See also [8] and [9].) [We have chosen the sign of $\Psi_{N}$ so that $\mathcal{A}_{N}^{\phi}$ will equal a sum of tree diagrams with propagators $1 / k^{2}$, where $k$ is the momentum. With this normalization, $\mathcal{A}_{N}^{\phi \text { tot }}$ equals $i(-1)^{N}$ times the amplitude defined using the usual field theory convention.]

For $N=5$, writing $z=z_{3}, w=z_{4}$, we have

$$
\mathcal{A}_{5}^{\phi}=-\frac{1}{4} \oint_{\mathcal{O}} \frac{z(1-w) d z d w}{(1-z) w f_{3} f_{4}}
$$

where

$$
\begin{aligned}
& f_{3}=k_{3} \cdot k_{2} z(z-w)+k_{3} \cdot k_{4} z(z-1)+k_{3} \cdot k_{5}(z-1)(z-w), \\
& f_{4}=k_{4} \cdot k_{2} w(w-z)+k_{4} \cdot k_{3} w(w-1)+k_{4} \cdot k_{5}(w-1)(w-z) .
\end{aligned}
$$

This is most conveniently evaluated using the global residue theorem. We write

$$
\mathcal{R}(\alpha, \beta)=-\lim _{\epsilon, \delta \rightarrow 0} \frac{1}{4} \oint_{\mathcal{O}_{\alpha \beta}} \frac{z(1-w) d z d w}{(1-\epsilon-z)(w-\delta) f_{3} f_{4}}
$$

for the residue of the integrand at $\alpha=\beta=0$, where $\mathcal{O}_{\alpha \beta}$ is an appropriately oriented contour about $\alpha=\beta=0 ; \alpha, \beta$ are chosen from the factors in the denominator of the integrand; and we have displaced the factors $1-z$ and $w$ by $\epsilon$ and $\delta$, respectively, in order to avoid singular configurations of these factors. Then

$$
\mathcal{A}_{5}^{\phi}=\mathcal{R}\left(f_{3}, f_{4}\right)=-\mathcal{R}\left(w, f_{4}\right)-\mathcal{R}(w, 1-z)-\mathcal{R}\left(f_{3}, 1-z\right)
$$

The term $\mathcal{R}(w, 1-z)$, for which the displacements by $\epsilon$ and $\delta$ are unnecessary, is easily evaluated:

$$
\mathcal{R}(w, 1-z)=-\frac{1}{4 k_{2} \cdot k_{3} k_{4} \cdot k_{5}}=-\frac{1}{s_{23} s_{45}},
$$

where we use the notation $s_{a b}=\left(k_{a}+k_{b}\right)^{2}, s_{a b c}=\left(k_{a}+k_{b}+k_{c}\right)^{2}$, etc. For the term $\mathcal{R}\left(w, f_{4}\right)$, we note that if $w=\delta, f_{3}=0$ implies $z=\mathcal{O}(\delta)$. Setting $w=x z$,

$$
\begin{array}{ll}
f_{3}=-z g_{3}+\mathcal{O}\left(\delta^{2}\right), & g_{3}=k_{3} \cdot k_{4}+k_{3} \cdot k_{5}(1-x) ; \\
f_{4}=-z g_{4}+\mathcal{O}\left(\delta^{2}\right), & g_{4}=k_{4} \cdot k_{3} x+k_{4} \cdot k_{5}(x-1) .
\end{array}
$$


Then

$$
\mathcal{R}\left(w, f_{4}\right)=-\frac{1}{4} \oint_{\mathcal{O}_{z g_{4}}} \frac{z d z z d x}{(1-z) x z^{3} g_{3} g_{4}}=-\frac{1}{4} \oint_{g_{4}=0} \frac{d x}{x g_{3} g_{4}}=-\frac{1}{s_{345}}\left[\frac{1}{s_{45}}+\frac{1}{s_{34}}\right] .
$$

Noting that the integrand of (3.4) is symmetric under the interchanges $z \leftrightarrow 1-w, k_{2} \leftrightarrow$ $k_{5}, k_{3} \leftrightarrow k_{4}, f_{3} \leftrightarrow f_{4}$, we deduce that

$$
\mathcal{R}\left(f_{3}, 1-z\right)=-\left[\frac{1}{s_{23}}+\frac{1}{s_{34}}\right] \frac{1}{s_{234}} .
$$

Putting together (3.9), (3.12) and (3.13), we see that $\mathcal{A}_{5}^{\phi}$ gives the sum of planar tree $\phi^{3}$ diagrams for momentum ordering $\left(k_{1}, k_{2}, k_{3}, k_{4}, k_{5}\right)$,

$$
\mathcal{A}_{5}^{\phi}=\frac{1}{s_{23} s_{234}}+\frac{1}{s_{34} s_{234}}+\frac{1}{s_{23} s_{45}}+\frac{1}{s_{345} s_{34}}+\frac{1}{s_{345} s_{45}} .
$$

This formula does not explicitly involve $k_{1}$ and hold when $k_{1}$ is off-shell, i.e. when $k_{1}^{2} \neq 0$, and the same is true of the form (3.3) for $\mathcal{A}_{4}^{\phi}$.

\section{Inductive proof for (partly) off-shell massless $\phi^{3}$ theory}

We shall prove by induction that (3.2) provides a formula for the sum of planar tree diagrams that is valid when $k_{1}$ is off-shell. We shall do this by establishing the recurrence relation

$$
\begin{aligned}
& A_{N}^{\phi}\left(k_{1}, k_{2}, \ldots, k_{N}\right)=\frac{1}{\bar{s}_{3}} A_{N-1}^{\phi}\left(k_{1}+k_{2}, \ldots, k_{N}\right) \\
& +\sum_{m=3}^{N-2} A_{m}^{\phi}\left(\pi_{m}, k_{2}, \ldots, k_{m}\right) \frac{1}{s_{m} \bar{s}_{m+1}} A_{N-m+1}^{\phi}\left(\bar{\pi}_{m+1}, k_{m+1}, \ldots, k_{N}\right) \\
& \quad+A_{N-1}^{\phi}\left(k_{N}+k_{1}, k_{2}, \ldots, k_{N-1}\right) \frac{1}{s_{N-1}},
\end{aligned}
$$

where

$$
\pi_{m}=-k_{2}-\ldots-k_{m}, \quad \bar{\pi}_{m}=-k_{m}-\ldots-k_{N}, \quad s_{m}=\pi_{m}^{2}, \quad \bar{s}_{m}=\bar{\pi}_{m}^{2},
$$

which holds if $A_{N}^{\phi}$ denotes the sum of planar tree diagrams with the first momentum offshell. This relation follows from the observation that, for $N>3$, the external line carrying the off-shell momentum $k_{1}$ enters the tree diagram either at a vertex in which one of the other two lines meeting there is internal or both are. In the former case, because the diagrams are planar with the given ordering of momenta, the other external line must either carry momentum $k_{2}$ or momentum $k_{N}$, and the internal line is a propagator carrying offshell momentum $k_{1}+k_{2}$ or $k_{1}+k_{N}$ joining it to an $(N-1)$-point tree amplitude in which all the other momenta are the remaining $k_{a}$ in order, and so on shell, providing the first and last terms in (4.1). In the latter case, the two off-shell internal lines have momenta $\pi_{m}$ and $\bar{\pi}_{m+1}$. 
To establish (4.1) holds for $A_{N}^{\phi}=\mathcal{A}_{N}^{\phi}$ defined by (3.2), we use the global residue theorem as we did in case $N=5$, again displacing the factors of $1-z_{3}$ and $z_{N-1}$ by $\epsilon$ and $\delta$, respectively, in order to avoid singular configurations

$$
\mathcal{A}_{N}^{\phi}=\frac{\varepsilon_{N}}{2^{N-3}} \oint_{\mathcal{O}} \frac{1}{\left(1-\epsilon-z_{3}\right)\left(z_{N-1}-\delta\right)} \prod_{a=3}^{N-2} z_{a} \prod_{a=4}^{N-1}\left(1-z_{a}\right) \prod_{b=5}^{N-1} \prod_{a=3}^{b-2}\left(z_{a}-z_{b}\right)^{2} \prod_{a=3}^{N-1} \frac{d z_{a}}{f_{a}} .
$$

Denoting the right hand side of (4.3) by $\mathcal{R}\left(f_{3}, f_{4}, \ldots, f_{N-2}, f_{N-1}\right)$, using the global residue theorem,

$$
\begin{aligned}
& \mathcal{R}\left(f_{3}, f_{4}, \ldots, f_{N-2}, f_{N-1}\right)=-\mathcal{R}\left(z_{N-1}^{\delta}, f_{4}, \ldots, f_{N-2}, f_{N-1}\right) \\
& \quad-\mathcal{R}\left(f_{3}, f_{4}, \ldots, f_{N-2}, 1-z_{3}^{\epsilon}\right)-\mathcal{R}\left(z_{N-1}^{\delta}, f_{4}, \ldots, f_{N-2}, 1-z_{3}^{\epsilon}\right),
\end{aligned}
$$

where $z_{3}^{\epsilon}=z_{3}+\epsilon$ and $z_{N-1}^{\delta}=z_{N-1}-\delta$. Applying the global residue theorem again,

$$
\begin{aligned}
\mathcal{R}\left(z_{N-1}^{\delta}, f_{4}, f_{5}, \ldots, f_{N-3}, f_{N-2}, 1-z_{3}^{\epsilon}\right)=-\mathcal{R}\left(z_{N-1}^{\delta}, f_{N-1}, f_{5}, \ldots, f_{N-3}, f_{N-2}, 1-z_{3}^{\epsilon}\right) \\
-\mathcal{R}\left(z_{N-1}^{\delta}, f_{4}, f_{5}, \ldots, f_{N-3}, f_{3}, 1-z_{3}^{\epsilon}\right)-\mathcal{R}\left(z_{N-1}^{\delta}, f_{N-1}, f_{5}, \ldots, f_{N-3}, f_{3}, 1-z_{3}^{\epsilon}\right), \\
\left.=(-1)^{N+1} \mathcal{R}\left(z_{N-1}^{\delta}, f_{5}, \ldots, f_{N-3}, f_{N-2}, f_{N-1}, 1-z_{3}^{\epsilon}\right)+\mathcal{R}\left(z_{N-1}^{\delta}, f_{3}, f_{4}, f_{5}, \ldots, f_{N-3}, 1-z_{3}^{\epsilon}\right)\right] \\
\quad+\mathcal{R}\left(z_{N-1}^{\delta}, f_{3}, f_{5}, \ldots, f_{N-3}, f_{N-1}, 1-z_{3}^{\epsilon}\right) .
\end{aligned}
$$

Repeating this process, we obtain

$$
\begin{aligned}
-\mathcal{R}\left(z_{N-1}^{\delta},\right. & \left.f_{4}, f_{5}, \ldots, f_{N-3}, f_{N-2}, 1-z_{3}^{\epsilon}\right) \\
& =(-1)^{N} \sum_{m=3}^{N-2} \mathcal{R}\left(z_{N-1}^{\delta}, f_{3}, f_{4}, \ldots, f_{m-1}, f_{m+2}, \ldots, f_{N-2}, f_{N-1}, 1-z_{3}^{\epsilon}\right) \\
& =\sum_{m=3}^{N-2} \mathcal{R}\left(f_{3}, f_{4}, \ldots, f_{m-1}, 1-z_{3}^{\epsilon}, z_{N-1}^{\delta}, f_{m+2}, \ldots, f_{N-2}, f_{N-1}\right) .
\end{aligned}
$$

After substituting this form for $\mathcal{R}\left(z_{N-1}^{\delta}, f_{4}, \ldots, f_{N-2}, 1-z_{3}^{\epsilon}\right)$ in (4.4), we shall show that the various terms correspond to the terms in the recurrence relation (4.1).

First consider $\mathcal{R}\left(z_{N-1}^{\delta}, f_{4}, \ldots, f_{N-2}, f_{N-1}\right)$ as $\delta \rightarrow 0$. As $z_{N-1}=\delta \rightarrow 0$ with $f_{a}=0,4 \leq a \leq N-1$, for general values of the external momenta satisfying the zero-mass condition, we must have that $z_{3}$ also vanishes, for otherwise we would have $k_{S}^{2}=0$, as in (2.18) with $s=N$, where $S$ is the set of $a$ for which $z_{a}$ vanishes as $\delta \rightarrow 0$ (including $N$ ), and we have stipulated that the $k_{a}$ are in general position. In fact the residue comes from the region where all $z_{a}=\mathcal{O}(\delta), 3 \leq a \leq N-1$. We shall first calculate the contribution from this region and then show it is necessary for all $z_{a}, 3 \leq a \leq N-1$, to vanish to get a non-zero contribution.

Set $z_{a}=x_{a} z_{3}, 3 \leq a \leq N$, so that $x_{3}=1, x_{N}=0$. Then, as in (2.15), for $3 \leq a \leq N-1$,

$$
f_{a}(z)=-z_{3}^{N-4} g_{a}(x)[1+\mathcal{O}(\delta)]
$$

where

$$
g_{a}(x)=\hat{g}_{a}(x) \prod_{\substack{c=3 \\ c \neq a}}^{N}\left(x_{a}-x_{c}\right)=\sum_{\substack{b=3 \\ b \neq a}}^{N} k_{a} \cdot k_{b} \prod_{\substack{c=3 \\ c \neq a, b}}^{N}\left(x_{a}-x_{c}\right)
$$


Then $\mathcal{R}\left(z_{N-1}^{\delta}, f_{4}, \ldots, f_{N-2}, f_{N-1}\right)$ is the residue of

$$
\begin{aligned}
-\frac{\varepsilon_{N-1}}{2^{N-3}} \oint \frac{d z_{3}}{z_{3} x_{N-1} g_{3}} & \prod_{a=4}^{N-2} x_{a} \prod_{b=5}^{N-1} \prod_{a=3}^{b-2}\left(x_{a}-x_{b}\right)^{2} \prod_{a=4}^{N-1} \frac{d x_{a}}{g_{a}} \\
& =-\frac{\varepsilon_{N-1}}{2^{N-3}} \oint \frac{1}{x_{N-1} g_{3}} \prod_{a=4}^{N-2} x_{a} \prod_{b=5}^{N-1} \prod_{a=3}^{b-2}\left(x_{a}-x_{b}\right)^{2} \prod_{a=4}^{N-1} \frac{d x_{a}}{g_{a}}
\end{aligned}
$$

at $g_{4}=\ldots=g_{N-1}=0$. In (4.9), in the limit $\delta \rightarrow 0$, a factor of $\delta^{(N-4)^{2}}$ in the numerator, coming from the products

$$
\prod_{a=3}^{N-2} z_{a} \prod_{b=5}^{N-1} \prod_{a=3}^{b-2}\left(z_{a}-z_{b}\right)^{2}
$$

in (4.3), is cancelled by an equal factor in the denominator, coming from

$$
\prod_{a=3}^{N-1} \frac{d z_{a}}{f_{a}}
$$

leaving a finite answer. As in (2.17),

$$
\sum_{a=3}^{N-1} x_{a} \hat{g}_{a}=\frac{\bar{s}_{3}}{2}
$$

So, when $g_{4}=\ldots=g_{N-1}=0$,

$$
g_{3}=\hat{g}_{3} \prod_{c=4}^{N}\left(x_{3}-x_{c}\right)=\frac{\bar{s}_{3}}{2} \prod_{c=4}^{N-1}\left(1-x_{c}\right),
$$

and, provided that there are no other contributions to this residue from other regions, $\mathcal{R}\left(z_{N-1}^{\delta}, f_{4}, \ldots, f_{N-2}, f_{N-1}\right)$ equals

$$
\begin{aligned}
-\frac{\varepsilon_{N-1}}{2^{N-4} \bar{s}_{3}} \oint \frac{1}{x_{N-1}\left(1-x_{4}\right)} \prod_{a=4}^{N-2} x_{a} & \prod_{a=5}^{N-1}\left(1-x_{a}\right) \prod_{b=6}^{N-1} \prod_{a=4}^{b-2}\left(x_{a}-x_{b}\right)^{2} \prod_{a=4}^{N-1} \frac{d x_{a}}{g_{a}} \\
& =-\frac{1}{\bar{s}_{3}} A_{N-1}^{\phi}\left(k_{1}+k_{2}, k_{3}, k_{4}, \ldots, k_{N-1}, k_{N}\right)
\end{aligned}
$$

by induction.

If we consider contributions to $\mathcal{R}\left(z_{N-1}^{\delta}, f_{4}, \ldots, f_{N-2}, f_{N-1}\right)$ from regions in which not all the $z_{a}, 3 \leq a \leq N-1$ vanish as $\delta \rightarrow 0$. As we have argued, $z_{3} \rightarrow 0$ as well as $z_{N-1}$ as $\delta \rightarrow 0$. If the set $S=\left\{a: z_{a} \rightarrow 0\right\}$ contains only $M$ of the $z_{a}, 3 \leq a \leq N$, where $M<N-2$, the product (4.11) in (4.3) will contribute a factor of $\delta^{(M-2)^{2}}$ to the denominator, but the product (4.10) will contribute a factor of $\delta^{L}$ where $L>(M-2)^{2}$, which implies that the contribution vanishes as $\delta \rightarrow 0$. This is because the products in (4.10) would contribute an exactly balancing contribution of $\delta^{(M-2)^{2}}$ if the $a \in S$ were consecutive but the second product in (4.10) contributes a factor of $\delta^{2}$ in the limit for every $a, b \in S$ with $3 \leq a<b \leq N-1$ and $b-a>1$, and this number is at a minimum for a 
given $M$ when the $a$ are consecutive. However, as $3, N-1 \in S$ and $M<N-2$, the $a \in S$ cannot be consecutive and so the contribution vanishes in the limit, establishing (4.14).

Because of the symmetry under $z_{a} \leftrightarrow 1-z_{N+2-a}, k_{a} \leftrightarrow k_{N+2-a}, 2 \leq a \leq N$, we have the corresponding result

$$
\mathcal{R}\left(f_{3}, f_{4}, \ldots, f_{N-2}, 1-z_{3}^{\epsilon}\right)=-A_{N-1}^{\phi}\left(k_{N}+k_{1}, k_{2}, \ldots, k_{N-1}\right) \frac{1}{s_{N-1}} .
$$

It remains to consider $\mathcal{R}\left(f_{3}, f_{4}, \ldots, f_{m-1}, 1-z_{3}^{\epsilon}, z_{N-1}^{\delta}, f_{m+2}, \ldots, f_{N-2}, f_{N-1}\right)$. This contribution comes from the region $z_{a}=\mathcal{O}(\delta), m+1 \leq a \leq N-1 ; 1-z_{a}=\mathcal{O}(\epsilon), 3 \leq a \leq$ $m$, for reasons similar to those that determined the appropriate regions for the residues already considered: if $S_{0}$ denotes the set of $a$ for which $z_{a} \rightarrow 0$ (including $a=N$ ) and $S_{1}$ denotes the set of $a$ for which $z_{a} \rightarrow 1$ (including $a=2$ ), then each of $S_{0}, S_{1}$ much contain one of $m$ or $m+1$ or we should have $k_{S_{0}}^{2}=0$ or $k_{S_{1}}^{2}=0$, contradicting the assumption that the $k_{a}$ are in general position. If $S_{0}$ does not consist of consecutive elements we should find again that the contribution will vanish in the limit and similarly for $S_{1}$. Thus the only way to get a non-zero contribution is to have $S_{0}=\{a: m+1 \leq a \leq N\}$ and $S_{1}=\{a: 2 \leq a \leq m\}$, which is the region we are considering.

Set $z_{a}=x_{a} z_{m+1}, m+1 \leq a \leq N ; 1-z_{a}=y_{a}\left(1-z_{m}\right), 2 \leq a \leq m$, so that $x_{m+1}=1, x_{N}=0$ and $y_{m}=1, y_{2}=0$. Then, as in (2.15), for $3 \leq a \leq m$,

$$
f_{a}(z)=(-1)^{m+1}\left(1-z_{m}\right)^{m-3} h_{a}(y)[1+\mathcal{O}(\delta, \epsilon)],
$$

where

$$
h_{a}(y)=\hat{h}_{a}(y) \prod_{\substack{c=2 \\ c \neq a}}^{m}\left(y_{a}-y_{c}\right) .=\sum_{\substack{b=2 \\ b \neq a}}^{m} k_{a} \cdot k_{b} \prod_{\substack{c=2 \\ c \neq a, b}}^{m}\left(y_{a}-y_{c}\right),
$$

and, for $m+1 \leq a \leq N-1$,

$$
f_{a}(z)=(-1)^{m+1} z_{m+1}^{N-m-2} g_{a}(x)[1+\mathcal{O}(\delta, \epsilon)]
$$

where

$$
g_{a}(x)=\hat{g}_{a}(x) \prod_{\substack{c=m+1 \\ c \neq a}}^{N}\left(x_{a}-x_{c}\right)=\sum_{\substack{b=m+1 \\ b \neq a}}^{N} k_{a} \cdot k_{b} \prod_{\substack{c=m+1 \\ c \neq a, b}}^{N}\left(x_{a}-x_{c}\right) .
$$

Then $\mathcal{R}\left(f_{3}, f_{4}, \ldots, f_{m-1}, 1-z_{3}^{\epsilon}, z_{N-1}^{\delta}, f_{m+2}, \ldots, f_{N-2}, f_{N-1}\right)$ is the residue at $g_{m+2}=$ $\ldots=g_{N-1}=0$ and $h_{3}=\ldots=h_{m-1}=0$ of

$$
\begin{aligned}
\frac{\varepsilon_{N} \varepsilon_{N, m}}{2^{N-3}} \int \prod_{a=3}^{m-1} \frac{d y_{a}}{h_{a}} & \prod_{a=3}^{m-2}\left(1-y_{a}\right)^{2} \prod_{a=4}^{m-1} y_{a} \prod_{a=3}^{m-3} \prod_{b=a+2}^{m-1}\left(y_{a}-y_{b}\right)^{2} \times \frac{d z_{m} d z_{m+1}}{\left(1-z_{3}\right) z_{N-1} h_{m} g_{m+1}} \\
& \times \prod_{a=m+2}^{N-1} \frac{d x_{a}}{g_{a}} \prod_{a=m+2}^{N-2} x_{a} \prod_{b=m+3}^{N-1}\left(1-x_{b}\right)^{2} \prod_{b=m+4}^{N-1} \prod_{a=m+2}^{b-2}\left(x_{a}-x_{b}\right)^{2},
\end{aligned}
$$

where $\varepsilon_{N, m}=(-1)^{(m+1) N}$. At $g_{m+2}=\ldots=g_{N-1}=0$, and $h_{3}=\ldots=h_{m-1}=0$, as in (2.17),

$$
g_{m+1}=\bar{s}_{m+1} \prod_{c=m+2}^{N-1}\left(1-x_{c}\right), \quad h_{m}=s_{m} \prod_{c=3}^{m-1}\left(1-y_{c}\right),
$$


so that (4.20) becomes

$$
\begin{aligned}
& -\int \frac{\varepsilon_{m}}{2^{m-3}\left(1-y_{3}\right) y_{m-1}} \prod_{a=3}^{m-1} \frac{d y_{a}}{h_{a}} \prod_{a=3}^{m-2}\left(1-y_{a}\right) \prod_{a=4}^{m-1} y_{a} \prod_{a=3}^{m-3} \prod_{b=a+2}^{m-1}\left(y_{a}-y_{b}\right)^{2} \frac{1}{s_{m}} \times \frac{d z_{m} d z_{m+1}}{\left(1-z_{m}\right) z_{m+1}} \\
& \times \frac{\varepsilon_{N-m+1}}{\bar{s}_{m+1} 2^{N-m-2}\left(1-x_{m+2}\right) x_{N-1}} \prod_{a=m+2}^{N-1} \frac{d x_{a}}{g_{a}} \prod_{a=m+2}^{N-2} x_{a} \prod_{b=m+3}^{N-1}\left(1-x_{b}\right) \prod_{b=m+4}^{N-1} \prod_{a=m+2}^{b-2}\left(x_{a}-x_{b}\right)^{2},(4.22)
\end{aligned}
$$

giving, on integration,

$$
A_{m}^{\phi}\left(\pi_{m}, k_{2}, \ldots, k_{m}\right) \frac{1}{s_{m} \bar{s}_{m+1}} A_{N-m+1}^{\phi}\left(\bar{\pi}_{m+1}, k_{m+1}, \ldots, k_{N}\right)
$$

as a contribution to the amplitude $A_{N}^{\phi}$, and, together with (4.14) and (4.15), establishing the recurrence relation (4.1).

\section{$5 \quad$ BCFW proof for massless $\phi^{3}$}

The proof given in section 4 that, with $\widehat{\Psi}_{N}$ constant, we obtain the tree amplitudes of massless $\phi^{3}$, would be difficult to extend to the gauge theory case, because the presence of four-point gluon vertices proliferates the number of terms involved in the induction. So, as a preliminary to discussing this case in section 6 , we now give a proof using the BCFW relations. To this end, choose a (possibly complex) momentum vector $\ell$ such that $\ell^{2}=\ell \cdot k_{2}=\ell \cdot k_{N}=0$, and consider

$$
A_{N}^{\phi}(\zeta)=A_{N}^{\phi}\left(k_{1}, k_{2}+\zeta \ell, k_{3}, \ldots, k_{N-1}, k_{N}-\zeta \ell\right),
$$

where $A_{N}^{\phi}$ denotes the sum of planar tree diagrams. Then $A_{N}^{\phi}(\zeta)$ is meromorphic in $\zeta$ with poles arising because of the propagators in the tree diagrams it comprises. The residues of these poles are given by the product of amplitudes $A_{m}^{\phi}$, where $m<N$ and so $A_{N}^{\phi}(\zeta)$ can be expressed as a sum over these residues, and this provides a recursion relation, which is the $\mathrm{BCFW}$ relation $[4,7]$.

If it were not the case that $A_{N}^{\phi}(\zeta) \rightarrow 0$ as $\zeta \rightarrow \infty$, a constant would have to be included, in essence a boundary term from infinity. This would indeed arise if we were considering the sum of all $N$-point tree functions regardless of the order of the external momenta, but, the ordering of momenta in $A_{N}^{\phi}$ and the fact that the displaced momenta $k_{2}+\zeta \ell$ and $k_{N}-\zeta \ell$ are not adjacent means that at least one propagator denominator in each tree diagram included in $A_{N}^{\phi}$ depends linearly on $\zeta$ and so $A_{N}^{\phi}(\zeta) \rightarrow 0$ as $\zeta \rightarrow \infty$.

Integrating around a small contour $\mathcal{O}_{\zeta}$ enclosing $\xi=\zeta$ and then moving the contour out to infinity,

$$
A_{N}^{\phi}(\zeta)=\oint_{\mathcal{O}_{\zeta}} \frac{A_{N}^{\phi}(\xi) d \xi}{\xi-\zeta}=-\sum_{i} \frac{\operatorname{Res}_{\zeta_{i}} A_{N}^{\phi}}{\zeta_{i}-\zeta},
$$

where the poles of $A_{N}^{\phi}(\zeta)$ occur at the points $\zeta=\zeta_{i}$. Define $\pi_{m}, \bar{\pi}_{m}, s_{m}, \bar{s}_{m}$ as in (4.2), and $\pi_{m}^{\zeta}$ and $\bar{\pi}_{m}^{\zeta}$ by replacing $k_{2}$ by $k_{2}^{\zeta}$ in $\pi_{m}$ and $k_{N}$ by $k_{N}^{\zeta}$ in $\bar{\pi}_{m}$, respectively, where

$$
k_{2}^{\zeta}=k_{2}+\zeta \ell, \quad k_{N}^{\zeta}=k_{N}-\zeta \ell .
$$


Then the poles of $A_{N}^{\phi}(\zeta)$ occur where $\left(\pi_{m}^{\zeta}\right)^{2}=0$ or $\left(\bar{\pi}_{m}^{\zeta}\right)^{2}=0$, i.e. at

$$
\zeta=s_{m} / 2 \pi_{m} \cdot \ell \equiv \zeta_{m}^{L}, \quad \text { and } \quad \zeta=-\bar{s}_{m} / 2 \bar{\pi}_{m} \cdot \ell \equiv \zeta_{m}^{R}, \quad 3 \leq m \leq N-1,
$$

with residues given by

$$
\operatorname{Res}_{\zeta_{m}^{L}} A_{N}^{\phi}=-A_{m}^{\phi}\left(\pi_{m}^{\zeta_{m}^{L}}, k_{2}^{\zeta_{m}^{L}}, k_{3}, \ldots, k_{m}\right) \frac{1}{2 \pi_{m} \cdot \ell} A_{N-m+2}^{\phi}\left(k_{1},-\pi_{m}^{\zeta_{m}^{L}}, k_{m+1}, \ldots, k_{N-1}, k_{N}^{\zeta_{m}^{L}}\right)
$$

and

$$
\operatorname{Res}_{\zeta_{m}^{R}} A_{N}^{\phi}=A_{m}^{\phi}\left(k_{1}, k_{2}^{\zeta_{m}^{R}}, k_{3}, \ldots, k_{m-1},-\bar{\pi}_{m}^{\zeta_{m}^{R}}\right) \frac{1}{2 \bar{\pi}_{m} \cdot \ell} A_{N-m+2}^{\phi}\left(\bar{\pi}_{m}^{\zeta_{m}^{R}}, k_{m}, \ldots, k_{N-1}, k_{N}^{\zeta_{m}^{R}}\right) .
$$

Then, putting $\zeta=0$ in (5.2),

$$
A_{N}^{\phi}\left(k_{1}, k_{2}, \ldots, k_{N}\right)=A_{N}^{\phi}(0)=-2 \sum_{m=3}^{N-1}\left[\frac{2 \pi_{m} \cdot \ell}{s_{m}} \operatorname{Res}_{\zeta_{m}^{L}} A_{N}^{\phi}-\frac{2 \bar{\pi}_{m} \cdot \ell}{\bar{s}_{m}} \operatorname{Res}_{\zeta_{m}^{R}} A_{N}^{\phi}\right] .
$$

This relation is sufficient to determine $A_{N}^{\phi}$ for $N>3$ from $A_{3}^{\phi}=1$. We now seek to show $A_{N}^{\phi}=\mathcal{A}_{N}^{\phi}$, as defined by (3.2) satisfies (5.7), with $\operatorname{Res}_{\zeta_{m}^{L}} A_{N}^{\phi}$ and $\operatorname{Res}_{\zeta_{m}^{R}} A_{N}^{\phi}$ given by (5.5) and (5.6), respectively.

Defining $\mathcal{A}_{N}^{\phi}(\zeta)$ analogously to $A_{N}^{\phi}(\zeta)$,

$$
\mathcal{A}_{N}^{\phi}(\zeta)=\frac{\varepsilon_{N}}{2^{N-3}} \oint \frac{1}{\left(1-z_{3}\right) z_{N-1}} \prod_{a=3}^{N-2} z_{a} \prod_{a=4}^{N-1}\left(1-z_{a}\right) \prod_{b=5}^{N-1} \prod_{a=3}^{b-2}\left(z_{a}-z_{b}\right)^{2} \prod_{a=3}^{N-1} \frac{d z_{a}}{f_{a}(z, \zeta)}
$$

where $f_{a}(z, \zeta)$ is obtained from $f_{a}(z)$ by $k_{2} \mapsto k_{2}^{\zeta}, k_{N} \mapsto k_{N}^{\zeta}$,

$$
f_{a}(z, \zeta)=\prod_{\substack{c=2 \\ c \neq a}}^{N}\left(z_{a}-z_{c}\right)\left[\frac{k_{a} \cdot\left(k_{2}+\zeta \ell\right)}{\left(z_{a}-1\right)}+\sum_{\substack{b=3 \\ b \neq a}}^{N-1} \frac{k_{a} \cdot k_{b}}{\left(z_{a}-z_{b}\right)}+\frac{k_{a} \cdot\left(k_{N}-\zeta \ell\right)}{z_{a}}\right]
$$

and the contour encloses the solutions of

$$
f_{a}(z, \zeta)=0, \quad 3 \leq a \leq N-1,
$$

for given $\zeta$, but excludes the poles at $z_{N-1}=0$ and $z_{3}=1$.

The poles of $\mathcal{A}_{N}^{\phi}(\zeta)$ occur when the contour is pinched between the zeros of the $f_{a}$ and either $z_{N-1}=0$ or $z_{3}=1$. First consider a solution of (5.10) which is such that, as $\zeta \rightarrow \zeta_{0}, z_{N-1} \rightarrow 0$. It may be that as $\zeta \rightarrow \zeta_{0}$ some other $z_{a} \rightarrow 0$. Suppose again that $S$ denotes the set of $a$, with $3 \leq a \leq N$, for which $z_{a} \rightarrow 0$. For essentially the same reasons used in section 4 to determine which contributions to the recurrence relation considered there were nonzero, having $z_{a} \rightarrow 0$ for $a \in S$ will not produce a pole unless the $a$ in $S$ are consecutive. Thus we must have $S=\{a: m \leq a \leq N\}$ for some $m$ with $3 \leq m \leq N-1$. Then, from (2.18), $k_{S}^{2}=\left(\bar{\pi}_{m}^{\zeta_{0}}\right)^{2}=0$, so that $\zeta_{0}=\zeta_{m}^{R}$, as defined by (5.4), and the solution to (5.10) has $z_{a} \rightarrow 0, m \leq a \leq N-1$, as $\zeta \rightarrow \zeta_{m}^{R}$. Similarly, there are solutions to (5.10) that have the property that $z_{a} \rightarrow 1,3 \leq a \leq m$, as $\zeta \rightarrow \zeta_{m}^{L}$, as defined by (5.4). 
We now investigate the (potential) pole at $\zeta=\zeta_{m}^{R}$, which comes from the region of integration in (5.8) given by

$$
z_{a}=\mathcal{O}(\delta), \quad m \leq a \leq N-1 .
$$

Set

$$
z_{a}=x_{a} z_{m}, m \leq a \leq N ; \quad \text { so } \quad x_{m}=1, x_{N}=0 .
$$

As in (2.15) and (4.7), for $m \leq a \leq N-1$,

$$
f_{a}(z, \zeta)=(-1)^{m} z_{m}^{N-m-1} g_{a}(x, \zeta) \prod_{c=2}^{m-1} z_{c}[1+\mathcal{O}(\delta)]
$$

where

$$
g_{a}(x, \zeta)=\hat{g}_{a}(x, \zeta) \prod_{\substack{c=m \\ c \neq a}}^{N}\left(x_{a}-x_{c}\right)=\sum_{\substack{b=m \\ b \neq a}}^{N} k_{a} \cdot k_{b}^{\zeta} \prod_{\substack{c=m \\ c \neq a, b}}^{N}\left(x_{a}-x_{c}\right) .
$$

As in (2.17) and (4.12),

$$
\sum_{a=m}^{N-1} x_{a} \hat{g}_{a}(x, \zeta)=\frac{1}{2}\left(\sum_{a=m}^{N} k_{a}^{\zeta}\right)^{2}=\left(\zeta-\zeta_{m}^{R}\right) \ell \cdot \bar{\pi}_{m}
$$

So when $g_{a}(z, \zeta)=0, \quad m+1 \leq a \leq N-1$,

$$
g_{m}(x, \zeta)=\left(\zeta-\zeta_{m}^{R}\right) \ell \cdot \bar{\pi}_{m} \prod_{c=m+1}^{N-1}\left(1-x_{c}\right)
$$

For $3 \leq a \leq m-1$,

$$
f_{a}(z, \zeta)=z_{a}^{N-m} h_{a}(z, \zeta)[1+\mathcal{O}(\delta)]
$$

where

$$
h_{a}(z, \zeta)=\sum_{\substack{b=2 \\ b \neq a}}^{m-1} k_{a} \cdot k_{b}^{\zeta} z_{a} \prod_{\substack{c=2 \\ c \neq a, b}}^{m-1}\left(z_{a}-z_{c}\right)-k_{a} \cdot \bar{\pi} \prod_{m}^{\zeta} \prod_{\substack{c=2 \\ c \neq a}}^{m-1}\left(z_{a}-z_{c}\right) .
$$

Noting $\varepsilon_{N}(-1)^{m(N+1)}=-\varepsilon_{m} \varepsilon_{N-m+2}$

$$
\begin{aligned}
& \operatorname{Res}_{\zeta_{m}^{R}} \mathcal{A}_{N}^{\phi}(\zeta)=\oint \frac{d \zeta}{2 \ell \cdot \bar{\pi}_{m}\left(\zeta-\zeta_{m}^{R}\right)} \\
& \quad \times \frac{\varepsilon_{m}}{2^{m-3}\left(1-z_{3}\right) z_{m-1}} \prod_{a=3}^{m-2} z_{a} \prod_{a=4}^{m-1}\left(1-z_{a}\right) \prod_{b=5}^{m-1} \prod_{a=3}^{b-2}\left(z_{a}-z_{b}\right)^{2} \prod_{a=3}^{m-1} \frac{d z_{a}}{h_{a}} \times\left[-\frac{d z_{m}}{z_{m}}\right] \\
& \quad \times \frac{\varepsilon_{N-m+2}}{2^{N-m-1}\left(1-x_{m+1}\right) x_{N-1}} \prod_{a=m+1}^{N-2} x_{a} \prod_{b=m+2}^{N-1}\left(1-x_{b}\right) \prod_{b=m+3 a=m+1}^{N-1} \prod_{a}^{b-2}\left(x_{a}-x_{b}\right)^{2} \prod_{a=m+1}^{N-1} \frac{d x_{a}}{g_{a}}
\end{aligned}
$$

Integrating with respect to $\zeta$ and $z_{m}$ shows that $\operatorname{Res}_{\zeta_{m}^{R}} \mathcal{A}_{N}^{\phi}$ provides the appropriate contribution to the $\mathrm{BCFW}$ relation,

$$
\operatorname{Res}_{\zeta_{m}^{R}} \mathcal{A}_{N}^{\phi}=\mathcal{A}_{m}^{\phi}\left(k_{1}, k_{2}^{\zeta_{m}^{R}}, k_{3}, \ldots, k_{m-1},-\bar{\pi}_{m}^{\zeta_{m}^{R}}\right) \frac{1}{2 \bar{\pi}_{m} \cdot \ell} A_{N-m+2}^{\phi}\left(\bar{\pi}_{m}^{\zeta_{m}^{R}}, k_{m}, \ldots, k_{N-1}, k_{N}^{\zeta_{m}^{R}}\right),
$$


since the pole at $z_{m}=0$ is exterior to the contour. The residue at $\zeta=\zeta_{m}^{L}$ follows similarly, using the symmetry under $m \leftrightarrow N+2-m, z_{a} \leftrightarrow 1-z_{a}, L \leftrightarrow R$, establishing the full BCFW relation and so proving the formula for $A_{N}^{\phi}$ by induction.

\section{$6 \quad$ Proof for pure gauge theory}

The CHY proposal $[1,5]$ for a choice of $\widehat{\Psi}_{N}(z ; k ; \epsilon)$ that gives the tree amplitudes for pure gauge theory in any space-time dimension employs the Pfaffian of the antisymmetric matrix

$$
\Psi_{N}(z ; k ; \epsilon)=\left(\begin{array}{cc}
A & D \\
C & B
\end{array}\right)
$$

where

$$
\begin{array}{llll}
A_{a b}=\frac{k_{a} \cdot k_{b}}{z_{a}-z_{b}}, & B_{a b}=\frac{\epsilon_{a} \cdot \epsilon_{b}}{z_{a}-z_{b}}, & C_{a b}=\frac{\epsilon_{a} \cdot k_{b}}{z_{a}-z_{b}}, a \neq b, & 1 \leq a, b \leq N ; \\
A_{a a}=B_{a a}=0, & C_{a a}=-\Sigma_{a}, & \Sigma_{a}=\sum_{\substack{c=1 \\
c \neq a}}^{N} \frac{\epsilon_{a} \cdot k_{c}}{z_{a}-z_{c}}, & 1 \leq a \leq N,
\end{array}
$$

$D_{a b}=-C_{b a}, 1 \leq a, b \leq N$. [Properties of Pfaffians are reviewed in appendix A, together with further some results needed here for our discussion.]

The Pfaffian of $\Psi_{N}(z ; k ; \epsilon)$ itself is zero when the conditions (1.1) hold, because then it has two null vectors arising from common null vectors of $A$ and $C$ : the vectors $(1,1, \ldots, 1)$ and $\left(z_{1}, z_{2}, \ldots, z_{N}\right)$. So, CHY consider the Pfaffian of the $(2 N-2) \times(2 N-2)$-dimensional matrix $\Psi_{N}^{(a, b)}(z ; k ; \epsilon)$ obtained by omitting the $a$-th and $b$-th rows and columns of $\Psi_{N}(z ; k ; \epsilon)$ and demonstrate that

$$
\operatorname{Pf}^{\prime} \Psi_{N}(z ; k ; \epsilon)=2 \frac{(-1)^{a+b}}{z_{a}-z_{b}} \operatorname{Pf} \Psi_{N}^{(a, b)}(z ; k ; \epsilon)
$$

is independent of $a, b$ for $1 \leq a<b \leq N$. Then they give evidence for the proposition that the tree amplitudes, $\mathcal{A}_{N}^{\mathrm{YM}}$, for gauge theory are given by taking

$$
\widehat{\Psi}_{N}(z ; k ; \epsilon)=-(-2)^{3-N}\left(\operatorname{Pf}^{\prime} \Psi_{N}(z ; k ; \epsilon)\right) \prod_{a=1}^{N}\left(z_{a}-z_{a+1}\right)
$$

in (1.7), where the normalization factor has been chosen for the convenience of the present discussion. The required Möbius invariance of $\widehat{\Psi}_{N}(z ; k ; \epsilon)$ is straightforward to verify.

As in (3.2),

$$
\mathcal{A}_{N}^{\mathrm{YM}}(k ; \epsilon)=\frac{\varepsilon_{N}}{2^{N-3}} \oint_{\mathcal{O}} \frac{\widetilde{\Psi}_{N}^{o}(z ; k ; \epsilon)}{\left(1-z_{3}\right) z_{N-1}} \prod_{a=3}^{N-2} z_{a} \prod_{a=4}^{N-1}\left(1-z_{a}\right) \prod_{b=5}^{N-1} \prod_{a=3}^{b-2}\left(z_{a}-z_{b}\right)^{2} \prod_{a=3}^{N-1} \frac{d z_{a}}{f_{a}},
$$

where again $\varepsilon_{N}=(-1)^{\frac{1}{2} N(N+1)}, f_{a}$ is given by (2.23), the contour $\mathcal{O}$ encircles the simultaneous solutions of $f_{a}(z, k)=0,3 \leq a \leq N-1$ (but excludes $z_{3}=1$ and $z_{N-1}=0$ ), and where

$$
\widetilde{\Psi}_{N}^{o}(z ; k ; \epsilon)=\lim _{z_{1} \rightarrow \infty}\left(z_{1}^{2} \mathrm{Pf}^{\prime} \Psi_{N}(z ; k ; \epsilon)\right) \prod_{a=2}^{N-1}\left(z_{a}-z_{a+1}\right) .
$$


Now,

$$
\lim _{z_{1} \rightarrow \infty} z_{1}^{2} \operatorname{Pf}^{\prime} \Psi_{N}(z ; k ; \epsilon)=\operatorname{Pf}^{\prime} \Psi_{N}^{o}(z ; k ; \epsilon),
$$

where $\Psi_{N}^{o}(z ; k ; \epsilon) \equiv \Psi^{o}$ is antisymmetric and

$$
\begin{aligned}
\Psi_{a b}^{o}(z) & =\Psi_{a b}(z), \quad \text { if } \quad a, b \neq 1, N+1 ; b \neq N+a \\
\Psi_{1 b}^{o} & =k_{1} \cdot k_{b}, \Psi_{N+1, N+b}^{o}=\epsilon_{1} \cdot \epsilon_{b}, 2 \leq b \leq N ; \quad \Psi_{a, N+a}^{o}=\sum_{c=2}^{N} \frac{\epsilon_{a} \cdot k_{c}}{z_{a}-z_{c}}, 2 \leq a \leq N ; \\
\Psi_{1, N+1}^{o} & =\sum_{c=2}^{N} z_{c} \epsilon_{1} \cdot k_{c} ; \quad \Psi_{1, N+b}^{o}=k_{1} \cdot \epsilon_{b}, 2 \leq b \leq N ; \quad \Psi_{a, N+1}^{o}=-\epsilon_{1} \cdot k_{a}, 2 \leq a \leq N ;
\end{aligned}
$$

and

$$
\operatorname{Pf}^{\prime} \Psi^{o}=\frac{(-1)^{a+b}}{z_{a}-z_{b}} \operatorname{Pf} \Psi_{(a b)}^{o}=(-1)^{1+a} \operatorname{Pf} \Psi_{(1 a)}^{o}
$$

independent of $a, b$ for $1<a<b \leq N, \Psi_{(a b)}^{o}$ being defined relative to $\Psi^{o}$ in the same way that $\Psi_{N}^{(a, b)}$ is relative to $\Psi_{N}$. Thus, in (6.6), (6.7),

$$
\widetilde{\Psi}_{N}^{o}(z ; k ; \epsilon)=(-1)^{N} \operatorname{Pf} \Psi_{N}^{o}(z ; k ; \epsilon)_{(2 N)} \prod_{a=1}^{N}\left(z_{a}-z_{a+1}\right) .
$$

We now give a proof that $\mathcal{A}_{N}^{\mathrm{YM}}$, defined by (6.6), does indeed give the $N$-gluon Yang-Mills tree amplitude by establishing that it satisfies a BCFW recurrence relation, as in (5.5)-(5.7). To this end, we need to consider $\mathcal{A}_{N}^{\mathrm{YM}}(\zeta)$, obtained from $\mathcal{A}_{N}^{\mathrm{YM}}$ by deforming the momenta $k_{2} \rightarrow k_{2}^{\zeta}=k_{2}+\zeta \ell, k_{N} \rightarrow k_{N}^{\zeta}=k_{N}-\zeta \ell$, as in (5.3), where, again, $\ell^{2}=\ell \cdot k_{2}=\ell \cdot k_{N}=0$, and also by deforming the basis of polarization vectors $\epsilon_{2}^{\zeta 1}, \epsilon_{2}^{\zeta 2}, \epsilon_{2}^{j}, 3 \leq j \leq d-2$, for $k_{2}^{\zeta}$, as in [7], so that

$$
\epsilon_{2}^{\zeta 1}+i \epsilon_{2}^{\zeta 2}=\bar{\ell}-2\left(\zeta / k_{2} \cdot k_{N}\right) k_{N}, \quad \epsilon_{2}^{\zeta 1}-i \epsilon_{2}^{\zeta 2}=\ell
$$

where we have introduced $\bar{\ell}$ such that $\bar{\ell}^{2}=\bar{\ell} \cdot k_{2}=\bar{\ell} \cdot k_{N}=0, \bar{\ell} \cdot \ell=2$, so that $\epsilon_{2}^{\zeta i} \cdot \epsilon_{2}^{\zeta j}=$ $\delta^{i j}, \epsilon_{2}^{\zeta j} \cdot k_{2}^{\zeta}=0,1 \leq i, j \leq N-2$, with $\epsilon_{2}^{\zeta j} \equiv \epsilon_{2}^{j}, 3 \leq j \leq N-2$, constant; and similarly for the basis of polarization vectors $\epsilon_{N}^{\zeta 1}, \epsilon_{N}^{\zeta 2}, \epsilon_{N}^{j}, 3 \leq j \leq d-2$, for $k_{N}^{\zeta}$.

So we now consider

$$
\mathcal{A}_{N}^{\mathrm{YM}}(\zeta)=\mathcal{A}_{N}^{\mathrm{YM}}\left(k_{1}, k_{2}^{\zeta}, \ldots, k_{N-1}, k_{N}^{\zeta} ; \epsilon_{1}, \epsilon_{2}^{\zeta}, \ldots, \epsilon_{N-1}, \epsilon_{N}^{\zeta}\right) .
$$

The difference between $\mathcal{A}_{N}^{\mathrm{YM}}(\zeta)$ here and $\mathcal{A}^{\phi}(\zeta)$ in section 5 is the factor of $\widetilde{\Psi}_{N}^{o}(z ; k ; \epsilon)$, as defined by (6.13), in the integrand of (6.6), and this does not affect the nature of the singularities in $\zeta$, which are thus still poles at $\zeta=\zeta_{m}^{L}, \zeta=\zeta_{m}^{R}, 3 \leq m \leq N-1$, as defined in (5.4). [The singularities of $\widetilde{\Psi}_{N}^{o}(z ; k ; \epsilon)$ occur when two or more of the $z_{a}$ coincide. When the $z_{a}$ are adjacent the calculation in appendix A shows that factors of $z_{a}-z_{a+1}$ cancel those in the denominator, so that $\widetilde{\Psi}_{N}^{o}(z ; k ; \epsilon)$ is regular there. If they are not adjacent, these are cancelled by the factors of $\left(z_{a}-z_{b}\right)^{2}, a>b+1$, as in section 4.] To demonstrate that $\mathcal{A}_{N}^{\mathrm{YM}}$ satisfies the BCFW recurrence relation we need to show that $\widetilde{\Psi}_{N}^{o}$ factorizes appropriately at these values of $\zeta$. 
The residue of the pole of $\mathcal{A}^{\phi}(\zeta)$ at $\zeta=\zeta_{m}^{R}$ comes from the region (5.11). So again we set $z_{a}=x_{a} z, m \leq a \leq N$, as in (5.12), so that $x_{m}=1, x_{N}=0$. Then, as $z_{m} \rightarrow 0, \Psi_{a b}^{o}(z)$, as defined by (6.9), is given by

$$
\begin{aligned}
& \Psi_{a b}^{o} \sim \frac{k_{a} \cdot k_{b}}{z_{a}-z_{b}}, \quad 2 \leq a<b<m ; \quad \Psi_{N+a, N+b}^{o} \sim \frac{\epsilon_{a} \cdot \epsilon_{b}}{z_{a}-z_{b}}, \quad 2 \leq a<b<m ; \\
& \Psi_{a b}^{o} \sim \frac{k_{a} \cdot k_{b}}{z_{a}}, \quad 2 \leq a<m \leq b \leq N ; \Psi_{N+a, N+b}^{o} \sim \frac{\epsilon_{a} \cdot \epsilon_{b}}{z_{a}}, \quad 2 \leq a<m \leq b \leq N ; \\
& \Psi_{a b}^{o} \sim \frac{k_{a} \cdot k_{b}}{z_{m}\left(x_{a}-x_{b}\right)}, m \leq a<b \leq N ; \quad \Psi_{N+a, N+b}^{o} \sim \frac{\epsilon_{a} \cdot \epsilon_{b}}{z_{m}\left(x_{a}-x_{b}\right)}, m \leq a<b \leq N ; \\
& \Psi_{a, N+b}^{o} \sim \frac{k_{a} \cdot \epsilon_{b}}{z_{a}-z_{b}}, \quad 2 \leq a, b<m, a \neq b ; \quad \Psi_{a, N+b}^{o} \sim-\frac{k_{a} \cdot \epsilon_{b}}{z_{b}}, \quad 2 \leq b<m \leq a \leq N ; \\
& \Psi_{a, N+b}^{o} \sim \frac{k_{a} \cdot \epsilon_{b}}{z_{a}}, \quad 2 \leq a<m \leq b \leq N ; \quad \Psi_{a, N+b}^{o} \sim \frac{k_{a} \cdot \epsilon_{b}}{z_{m}\left(x_{a}-x_{b}\right)}, m \leq a, b \leq N, a \neq b ; \\
& \Psi_{a, N+a}^{o} \sim \hat{\Sigma}_{a}, \quad 2 \leq a<m ; \quad \Psi_{a, N+a}^{o} \sim \frac{1}{z_{m}} \hat{\Sigma}_{a}, \quad m \leq a \leq N .
\end{aligned}
$$

where

$$
\begin{aligned}
& \hat{\Sigma}_{1}=\sum_{c=2}^{m-1} z_{c} \epsilon_{1} \cdot k_{c} ; \quad \hat{\Sigma}_{a}=\sum_{\substack{c=2 \\
c \neq a}}^{m-1} \frac{\epsilon_{a} \cdot k_{c}}{z_{a}-z_{c}}+\sum_{c=m}^{N} \frac{\epsilon_{a} \cdot k_{c}}{z_{a}}, 2 \leq a<m ; \\
& \hat{\Sigma}_{a}=\sum_{\substack{c=m \\
c \neq a}}^{N} \frac{\epsilon_{a} \cdot k_{c}}{x_{a}-x_{c}}, m \leq a \leq N .
\end{aligned}
$$

Now rearrange the columns of $\Psi_{a b}^{o}$ by defining $u(a)=a$ for $1 \leq a<m$ or $N+m \leq$ $a \leq 2 N ; u(a)=N+a-m+1$ for $m \leq a \leq 2 m-2 ; u(a)=N+a-m+1$ for $2 m-2<a \leq N+m-2$; and set

$$
\Theta_{a b}=\Psi_{u(a) u(b)}^{o}
$$

so that $\Theta_{a b}=-\Theta_{b a}$. Then

$$
\Theta=\left[\begin{array}{cc}
E & F \\
-F^{T} & z_{m}^{-1} G
\end{array}\right],
$$

where

$$
\begin{aligned}
& E=\Psi_{m}^{o}\left(k_{1}, \ldots, k_{m-1},-\bar{\pi}_{m} ; \epsilon_{1}, \ldots, \epsilon_{m-1}, \epsilon^{s} ; z_{3}, \ldots, z_{m-1}\right)_{(m, 2 m)} \\
& G=\Psi_{N-m+2}^{o}\left(\bar{\pi}_{m}, k_{m}, \ldots, k_{N} ; \epsilon^{s}, \epsilon_{m}, \ldots, \epsilon_{N} ; x_{m+1}, \ldots, x_{N-1}\right)_{(1, N-m+3)}
\end{aligned}
$$

where $\bar{\pi}_{m}=-k_{m}-\ldots-k_{N}$, as in $(4.2)$, and $F_{a b}=\alpha_{a} \cdot \beta_{b}$, with

$$
\begin{aligned}
& \alpha_{1}=k_{1}, \quad \alpha_{a}=\frac{k_{a}}{z_{a}}, 2 \leq a<m, \quad \alpha_{m}=\epsilon_{1}, \quad \alpha_{a}=\frac{\epsilon_{a}}{z_{a}}, m<a \leq 2 m-2, \\
& \beta_{b}=k_{b}, 1 \leq b \leq N-m+2, \quad \beta_{b}=\epsilon_{b}, N-m+3 \leq b \leq 2 N-2 m+4
\end{aligned}
$$

[Note that $\epsilon_{s}, \bar{\pi}_{m}$ do not appear directly in the expressions for $E, G$ because of the rows omitted in $\left[\Psi_{m}^{o}\right]_{(m, 2 m)}$ and $\left[\Psi_{N-m+2}^{o}\right]_{(1, N-m+3)}$, respectively. $]$ 
Now, it is $\Psi_{(2, N)}^{o}$ that we want to consider rather than $\Psi^{o}$; replacing $\Psi^{o}$ with $\Psi_{(2, N)}^{o}$ effectively replaces $\Theta$ with

$$
\Theta_{(2, N+m-1)}=\left[\begin{array}{cc}
E_{(2)} & F_{[2,1]} \\
-F_{[2,1]}^{T} & z_{m}^{-1} G_{(1)}
\end{array}\right],
$$

where $E_{(a)}$ indicates that the $a$-th row and column have been removed from $E$, and similarly for $G_{(a)}$, and $F_{[a, b]}$ indicates that the $a$-th row and $b$-th column have been removed from $F$.

$$
\operatorname{Pf} \Psi_{(2 N)}^{o}=\operatorname{Pf} \Theta_{(2, N+m-1)}
$$

So, using the Factorization Lemma of appendix A, as $z_{m} \rightarrow 0$,

$$
\operatorname{Pf} \Phi(z) \sim z_{m}^{-(N-m)} \sum_{r} \operatorname{Pf} \widetilde{E}^{r} \times \operatorname{Pf} \widetilde{G}^{r}
$$

where

$$
\widetilde{E}^{r}=\left[\begin{array}{cc}
E_{(2)} & \left(\alpha_{[2]}^{r}\right)^{T} \\
-\alpha_{[2]}^{r} & 0
\end{array}\right], \quad \widetilde{G}^{r}=\left[\begin{array}{cc}
0 & \left(\beta_{[1]}^{r}\right)^{T} \\
-\beta_{[1]}^{r} & G_{(1)}
\end{array}\right],
$$

where $\alpha^{r}=\alpha \cdot \epsilon^{r}, \beta^{r}=\beta \cdot \epsilon^{r}$ and $\alpha_{[a]}^{r}, \beta_{[a]}^{r}$ denote $\alpha^{r}, \beta^{r}$, respectively, omitting the $a$-th entry. Then

$$
\widetilde{E}^{r}=\Psi_{m}^{o}\left(k_{1}, \ldots, k_{m-1},-\bar{\pi}_{m} ; \epsilon_{1}, \ldots, \epsilon_{m-1}, \epsilon^{s} ; z_{3}, \ldots, z_{m-1}\right)_{(2, m)}
$$

and, if we move the first row and column into the $(N-m+1)$-th positions, $\widetilde{G}^{r}$ becomes

$$
\Psi_{N-m+2}^{o}\left(\bar{\pi}_{m}, k_{m}, \ldots, k_{N} ; \epsilon^{s}, \epsilon_{m}, \ldots, \epsilon_{N} ; x_{m+1}, \ldots, x_{N-1}\right)_{(1, N-m+2)} .
$$

In principle, in (6.26), $\epsilon^{r}$ should run over the $d$ elements of a basis for the whole space, but we can restrict to a basis for the polarization states for $\bar{\pi}_{m}$, since the metric tensor is

$$
\eta_{\mu \nu}=\sum_{r} \epsilon_{\mu}^{r} \epsilon_{\nu}^{r}+\frac{1}{\bar{\pi}_{m} \cdot k}\left(k_{\mu} \bar{\pi}_{m \nu}+\bar{\pi}_{m \mu} k_{\nu}\right)
$$

where $k$ is chosen so that $k \cdot \epsilon_{r}=k^{2}=0, k \cdot \bar{\pi}_{m} \neq 0$, and the contributions from the last two terms vanish because

$$
\begin{aligned}
& \operatorname{Pf} \Psi_{m}^{o}\left(k_{1}, \ldots, k_{m-1},-\bar{\pi}_{m} ; \epsilon_{1}, \ldots, \epsilon_{m-1}, \bar{\pi}_{m} ; z_{3}, \ldots, z_{m-1}\right)_{(2, m)} \\
& \quad=\operatorname{Pf} \Psi_{N-m+2}^{o}\left(\bar{\pi}_{m}, k_{m}, \ldots, k_{N} ; \bar{\pi}_{m}, \epsilon_{m}, \ldots, \epsilon_{N} ; x_{m+1}, \ldots, x_{N-1}\right)_{(1, N-m+2)}=0,
\end{aligned}
$$

using, in the first case, that $\Psi_{m(2, m)}^{o}$ has null vector $\left(1, z_{3}-1, \ldots, z_{m-1}-1,0, \ldots, 0,1\right)$ and, in the second case, that $\Psi_{N-m+2(1, N-m+2)}^{o}$ has null vector $\left(1, x_{m+1}, \ldots, x_{N-1}, 0,1,0, \ldots, 0\right)$. As $z_{m} \rightarrow 0$,

$$
\prod_{a=2}^{N-1}\left(z_{a}-z_{a+1}\right) \rightarrow z_{m-1} z_{m}^{N-m} \prod_{a=2}^{m-2}\left(z_{a}-z_{a+1}\right) \prod_{a=m}^{N-1}\left(x_{a}-x_{a+1}\right),
$$

implying the desired factorization property as $z_{m} \rightarrow 0$,

$$
\Psi_{N}^{o}\left(k_{1}, \ldots, k_{N} ; \epsilon_{1}, \ldots, \epsilon_{N} ; z_{3}, \ldots, z_{N-1}\right)_{(2, N)}
$$




$$
\begin{aligned}
\sim \sum_{s} \Psi_{m}^{o} & \left.k_{1}, \ldots, k_{m-1},-\bar{\pi}_{m} ; \epsilon_{1}, \ldots, \epsilon_{m-1}, \epsilon^{s} ; z_{3}, \ldots, z_{m-1}\right)_{(2, m)} \\
& \times \Psi_{N-m+2}^{o}\left(\bar{\pi}_{m}, k_{m}, \ldots, k_{N} ; \epsilon^{s}, \epsilon_{m}, \ldots, \epsilon_{N} ; x_{m+1}, \ldots, x_{N-1}\right)_{(1, N-m+2)}
\end{aligned}
$$

This, together with the corresponding result for the pole at $\zeta=\zeta_{m}^{R}$, which holds by symmetry, establishes the BCFW recurrence relation.

\section{Acknowledgments}

We are grateful to Nima Arkani-Hamed and Song He for discussions. LD thanks the Institute for Advanced Study at Princeton for its hospitality. LD was partially supported by the U.S. Department of Energy, Grant No. DE-FG02-06ER-4141801, Task A, and PG was partially supported by NSF grant No. PHY-1314311.

\section{A Properties of Pfaffians}

The Pfaffian $\operatorname{Pf} A$ is defined for an antisymmetric matrix $A=-A^{T}$ by $\operatorname{det} A=(\operatorname{Pf} A)^{2}$, subject to a choice of $\operatorname{sign}$. If $\operatorname{dim} A$ is odd, $\operatorname{Pf} A=\operatorname{det} A=0$; if $\operatorname{dim} A$ is even, $\operatorname{Pf} A$ is a rational function of the coefficients of $A$. Explicitly, if $\operatorname{dim} A=M=2 N$,

$$
\operatorname{Pf} A=\sum_{\rho \in \mathfrak{S}_{2 N}} \frac{1}{2^{N} N !} \operatorname{sign} \rho \prod_{i=1}^{N} a_{\rho(2 i-1) \rho(2 i)},
$$

where $\mathfrak{S}_{M}$ denotes the group of permutations of $1,2, \ldots, M$.

We can write $\operatorname{Pf} A$ as a sum over the pairings $\varrho=\left\{\left(i_{1}, j_{1}\right),\left(i_{2}, j_{2}\right), \ldots,\left(i_{N}, j_{N}\right)\right\}$ of the integers $1,2, \ldots, 2 N-1,2 N$. Denote the set of such pairings by $\mathfrak{P}_{N}$. We can choose a unique description of the labeling of the pairing by specifying that $i_{a}<j_{a}$ and $i_{a}<i_{b}$ if $1 \leq a<b \leq N$. Given $\varrho \in \mathfrak{P}_{N}$, define $\rho_{\varrho} \in \mathfrak{S}_{2 N}$ by $\rho_{\varrho}(2 a-1)=i_{a}, \rho_{\varrho}(2 a)=j_{a}, 1 \leq a \leq N$, and set $\operatorname{sign} \varrho=\operatorname{sign} \rho_{\varrho}$. Then

$$
\operatorname{Pf} A=\sum_{\varrho \in \mathfrak{P}_{N}} \text { sign } \varrho \prod_{i=1}^{N} a_{i_{a} j_{a}} .
$$

If $A_{(i j)}$ denotes the matrix obtained from $A$ by deleting the $i$-th and $j$-th rows and $i$-th and $j$-th columns. Then $A_{(i j)}$ is an antisymmetric matrix with $\operatorname{dim} A_{(i j)}=\operatorname{dim} A-2$. Then we have the expansion

$$
\operatorname{Pf} A=\sum_{j=2}^{M}(-1)^{j} a_{1 j} \operatorname{Pf} A_{(1 j)} .
$$

In order to establish the factorization result in section 6 , we need a result on the factorization of Pfaffians in suitable limits, which we prove here. First we establish a preliminary result, which may serve to elucidate the subsequent argument. 
Preliminary Lemma. Suppose $E, G$ are antisymmetric matrices of dimensions of $m \times m$ and $n \times n$, respectively; $a, b$ are vectors of dimensions $m$ and $n$, respectively; and $F=a b^{T}$, i.e. $F_{i j}=a_{i} b_{j}$. Let

$$
\Phi=\left[\begin{array}{cc}
E & F \\
-F^{T} & G
\end{array}\right] .
$$

Then, if $m+n$ is odd, $\operatorname{Pf} \Phi=0$; if $m+n, m$ and $n$ are even, $\operatorname{Pf} \Phi=\operatorname{Pf} E \times \operatorname{Pf} G$; if $m+n$ is even, and $m$ and $n$ are odd, $\operatorname{Pf} \Phi=\operatorname{Pf} \widetilde{E} \times \operatorname{Pf} \widetilde{G}$, where

$$
\widetilde{E}=\left[\begin{array}{cc}
E & a^{T} \\
-a & 0
\end{array}\right], \quad \widetilde{G}=\left[\begin{array}{cc}
0 & b^{T} \\
-b & G
\end{array}\right] .
$$

Proof. Suppose $m+n$ even, $m+n=2 N$.

$$
\operatorname{Pf} \Phi=\sum_{\varrho \in \mathfrak{P}_{N}} \operatorname{sign} \varrho \prod_{a=1}^{N} \Phi_{i_{a} j_{a}},
$$

where $\varrho=\left\{\left(i_{1}, j_{1}\right),\left(i_{2}, j_{2}\right), \ldots,\left(i_{N}, j_{N}\right)\right\}$. Let $C_{\varrho}$ denote the number of $a$ for which $i_{a} \leq m$ and $j_{a}>m . m$ and $n$ are either both odd or both even and $C_{\varrho}$ has to be odd if $m, n$ are odd, and even if $m, n$ are even.

For each $\varrho \in \mathfrak{P}_{N}$ with a given value of $C_{\varrho}>1$, we associate to $\varrho^{\prime} \in \mathfrak{P}_{N}$, which is obtained by replacing the pairs $\left(i_{c}, j_{c}\right),\left(i_{d}, j_{d}\right)$, where $c, d$ are the largest values of $a$ such that $i_{a} \leq m$ and $j_{a}>m$, by the pairs $\left(i_{c}, j_{d}\right),\left(i_{d}, j_{c}\right)$. Then sign $\varrho=-\operatorname{sign} \varrho^{\prime}$, but the value of the product

$$
\prod_{a=1}^{N} \Phi_{i_{a} j_{a}}
$$

is the same for $\varrho^{\prime}$ as for $\varrho$. Thus the contributions to the sum over $\varrho \in \mathfrak{P}_{N}$ from those $\varrho$ with $C_{\varrho}>1$ cancel in pairs. Since $C_{\varrho}$ has the same parity as $m$ and $n$, all the nonzero contributions come from $C_{\varrho}=0$ if $m, n$ are even and from $C_{\varrho}=1$ if $m, n$ are odd.

If $m, n$ are even, each pairing $\varrho \in \mathfrak{P}_{N}$ making a nonzero contribution to $\operatorname{Pf} \Phi$ is the union of parings $\varrho_{1} \in \mathfrak{P}_{\frac{1}{2} m}$, of $1,2, \ldots, m$, and $\varrho_{2} \in \mathfrak{P}_{\frac{1}{2} n}$, of $m+1,2, \ldots, m+n$. So, the sum over such $\varrho \in \mathfrak{P}_{N}$ is the product of sums over $\varrho_{1} \in \mathfrak{P}_{\frac{1}{2} m}$ and over $\varrho_{2} \in \mathfrak{P}_{\frac{1}{2} n}$, with $\operatorname{sign} \varrho=\operatorname{sign} \varrho_{1} \operatorname{sign} \varrho_{2}$. The product (A.7) factors into two products corresponding to the pairings $\varrho_{1}$ and $\varrho_{2}$, respectively. Thus $\operatorname{Pf} \Phi=\operatorname{Pf} E \times \operatorname{Pf} G$.

If $m, n$ are odd, each $\varrho \in \mathfrak{P}_{N}$ making a nonzero contribution to $\operatorname{Pf} \Phi$ involves just one pair $\left(i_{0}, j_{0}\right)$ with $i_{0} \leq m$ and $j_{0}>m$. The remaining pairs in $\varrho$ are the union of parings $\varrho_{1}^{\prime} \in \mathfrak{P}_{\frac{1}{2}(m-1)}$, of $\left\{i: 1 \leq i \leq m, i \neq i_{0}\right\}$, and $\varrho_{2}^{\prime} \in \mathfrak{P}_{\frac{1}{2}(n-1)}$, of $\left\{j: m<j \leq m+n, j \neq j_{0}\right\}$. Define the pairing $\varrho_{1} \in \mathfrak{P}_{\frac{1}{2}(m+1)}$, of $\left\{i: 1 \leq i \leq m ; i_{*}\right\}$, by appending $\left(i_{0}, i_{*}\right)$ to $\varrho_{1}^{\prime}$, and the pairing $\varrho_{2} \in \mathfrak{P}_{\frac{1}{2}(n+1)}$, of $\left\{j: j_{*} ; m<j \leq m+n\right\}$, by appending $\left(j_{*}, j_{0}\right)$ to $\varrho_{2}^{\prime}$. Then the product (A.7) for $\varrho$ factors into products associated with $\varrho_{1}^{\prime}$ and $\varrho_{2}^{\prime}$, together with

$$
\Phi_{i_{0} j_{0}}=F_{i_{0} j_{0}}=a_{i_{0}} b_{j_{0}}=\widetilde{E}_{i_{0} i_{*}} \widetilde{G}_{j_{*} j_{0}},
$$

labeling the last row and column of $\widetilde{E}$ by $i_{*}$ and the first row and column of $\widetilde{G}$ by $j_{*}$, and, again, sign $\varrho=\operatorname{sign} \varrho_{1} \operatorname{sign} \varrho_{2}$. The sum over $\varrho \in \mathfrak{P}_{N}$ corresponds to the product of sums over $\varrho_{1} \in \mathfrak{P}_{\frac{1}{2}(m+1)}$ and $\varrho_{2} \in \mathfrak{P}_{\frac{1}{2}(n+1)}$, yielding $\operatorname{Pf} \Phi=\operatorname{Pf} \widetilde{E} \times \operatorname{Pf} \widetilde{G}$.

Now we proceed to prove the result needed in section 6 . 
Factorization Lemma. Again suppose $E, G$ are antisymmetric matrices of dimensions of $m \times m$ and $n \times n$, respectively; but now suppose that $a_{i}=\left(a_{i}^{\alpha}\right), 1 \leq i \leq m, b_{j}=\left(b_{j}^{\alpha}\right), 1 \leq$ $j \leq n$, are $d$-dimensional vectors and $F_{i j}=a_{i} \cdot b_{j}$ and consider the leading behavior of

$$
\Phi(z)=\left[\begin{array}{cc}
E & F \\
-F^{T} & z^{-1} G
\end{array}\right], \quad \text { as } \quad z \rightarrow 0 .
$$

If $m+n$ is odd, $\operatorname{Pf} \Phi(z)=0$; if $m+n, m, n$ are even, $\operatorname{Pf} \Phi(z) \sim z^{-\frac{1}{2} n} \operatorname{Pf} E \times \operatorname{Pf} G$, as $z \rightarrow 0$; if $m+n$ is even, and $m$ and $n$ are odd,

$$
\operatorname{Pf} \Phi(z) \sim z^{-\frac{1}{2}(n-1)} \sum_{\alpha} \operatorname{Pf} \widetilde{E}^{\alpha} \times \operatorname{Pf} \widetilde{G}^{\alpha} \quad \text { as } z \rightarrow 0
$$

where

$$
\widetilde{E}^{\alpha}=\left[\begin{array}{cc}
E & \left(a^{\alpha}\right)^{T} \\
-a^{\alpha} & 0
\end{array}\right], \quad \widetilde{G}^{\alpha}=\left[\begin{array}{cc}
0 & \left(b^{\alpha}\right)^{T} \\
-b^{\alpha} & G
\end{array}\right] .
$$

Proof. Suppose $m+n$ even, $m+n=2 N$. With $\varrho=\left\{\left(i_{1}, j_{1}\right),\left(i_{2}, j_{2}\right), \ldots,\left(i_{N}, j_{N}\right)\right\}$, the contribution

$$
\operatorname{sign} \varrho \prod_{a=1}^{N} \Phi(z)_{i_{a} j_{a}},
$$

to $\operatorname{Pf} \Phi(z)$ is $\mathcal{O}\left(z^{-M_{\varrho}}\right)$, where $M_{\varrho}$ is the number of $a$ for which $i_{a}>m$, for then $\Phi(z)_{i_{a} j_{a}}=\mathcal{O}\left(z^{-1}\right)$, but $\Phi(z)_{i_{a} j_{a}}=\mathcal{O}(1)$ otherwise. If $C_{\varrho}$ again denotes the number of $a$ for which $i_{a} \leq m$ and $j_{a}>m, M_{\varrho}=\frac{1}{2}\left(n-C_{\varrho}\right)$, so the behavior of $\operatorname{Pf} \Phi(z)$ as $z \rightarrow 0$ is dominated by terms with $C_{\varrho}$ as small as possible, i.e. $C_{\varrho}=0$ for $m, n$ even and $C_{\varrho}=1$ for $m, n$ odd. So, reasoning as in Lemma 1 , if $m, n$ are even, the leading contributions come from $\varrho \in \mathfrak{P}_{N}$ which are the union of parings $\varrho_{1} \in \mathfrak{P}_{\frac{1}{2} m}$, of $1,2, \ldots, m$, and $\varrho_{2} \in \mathfrak{P}_{\frac{1}{2} n}$, of $m+1,2, \ldots, m+n$, and $\operatorname{Pf} \Phi(z) \sim z^{-\frac{1}{2} n} \operatorname{Pf} E \times \operatorname{Pf} G$, as $z \rightarrow 0$.

If $m, n$ are odd, each $\varrho \in \mathfrak{P}_{N}$ making a leading contribution to $\operatorname{Pf} \Phi$ involves just one pair $\left(i_{0}, j_{0}\right)$ with $i_{0} \leq m$ and $j_{0}>m$. As in Lemma 1 , the remaining pairs in $\varrho$ are the union of parings $\varrho_{1}^{\prime} \in \mathfrak{P}_{\frac{1}{2}(m-1)}$, of $\left\{i: 1 \leq i \leq m, i \neq i_{0}\right\}$, and $\varrho_{2}^{\prime} \in \mathfrak{P}_{\frac{1}{2}(n-1)}$, of $\left\{j: m<j \leq m+n, j \neq j_{0}\right\}$. So again, define the pairing $\varrho_{1} \in \mathfrak{P}_{\frac{1}{2}(m+1)}$, of $\left\{i: 1 \leq i \leq m ; i_{*}\right\}$, by appending $\left(i_{0}, i_{*}\right)$ to $\varrho_{1}^{\prime}$, and the pairing $\varrho_{2} \in \mathfrak{P}_{\frac{1}{2}(n+1)}$, of $\left\{j: j_{*} ; m<j \leq m+n\right\}$, by appending $\left(j_{*}, j_{0}\right)$ to $\varrho_{2}^{\prime}$. Then the product (A.12) for $\varrho$ factors into products associated with $\varrho_{1}^{\prime}$ and $\varrho_{2}^{\prime}$, together with

$$
\Phi_{i_{0} j_{0}}=F_{i_{0} j_{0}}=\sum_{\alpha} a_{i_{0}}^{\alpha} b_{j_{0}}^{\alpha}=\sum_{\alpha} \widetilde{E}_{i_{0} i_{*}}^{\alpha} \widetilde{G}_{j_{*} j_{0}}^{\alpha}
$$

labeling the last row and column of $\widetilde{E}$ by $i_{*}$ and the first row and column of $\widetilde{G}$ by $j_{*}$, and, again, sign $\varrho=\operatorname{sign} \varrho_{1} \operatorname{sign} \varrho_{2}$. The sum over $\varrho \in \mathfrak{P}_{N}$ corresponds to the product of sums over $\varrho_{1} \in \mathfrak{P}_{\frac{1}{2}(m+1)}$ and $\varrho_{2} \in \mathfrak{P}_{\frac{1}{2}(n+1)}$, yielding

$$
\operatorname{Pf} \Phi(z) \sim z^{-\frac{1}{2}(n-1)} \sum_{\alpha} \operatorname{Pf} \widetilde{E}^{\alpha} \times \operatorname{Pf} \widetilde{G}^{\alpha} \quad \text { as } z \rightarrow 0 .
$$




\section{B Massive $\phi^{3}$ theory}

In this appendix, we show how the results for tree amplitudes in massless $\phi^{3}$ theory, established in sections 2 to 5 , can be extended to the massive case by replacing $\hat{f}_{a}$, defined by (1.1), by $\breve{f}_{a}$, defined as in (1.8),

$$
\check{f}_{a}(z, k)=\sum_{\substack{b \in A \\ b \neq a}} \frac{k_{a} \cdot k_{b}}{z_{a}-z_{b}}-\frac{a_{0}}{\left(z_{a}-z_{a+1}\right)}-\frac{a_{0}}{\left(z_{a}-z_{a-1}\right)}, \quad a \in A .
$$

As in section 2, suppose $z_{a} \rightarrow z_{S}$ for $z \in S \subset A$, with $z_{a}=z_{S}+\epsilon x_{a}+\mathcal{O}\left(\epsilon^{2}\right)$, as $\epsilon \rightarrow 0$, and $z_{a} \not \rightarrow z_{S}$ for $z \notin S$. Then, in place of (2.14) and (2.15), we have

$$
\begin{aligned}
& \breve{f}_{a}(z, k)=\frac{1}{\epsilon} \breve{g}_{a}(x, k)[1+\mathcal{O}(\epsilon)], \quad \breve{g}_{a}(x, k)=\sum_{\substack{b \in S \\
b \neq a}} \frac{k_{a} \cdot k_{b}}{x_{a}-x_{b}}-\sum_{\substack{b \in S \\
b=a \pm 1}} \frac{a_{0}}{x_{a}-x_{b}}, \quad a \in S, \\
& \breve{f}_{a}(z, k)=\sum_{b \in S} \frac{k_{a} \cdot k_{b}}{z_{a}-z_{S}}-\sum_{\substack{b \in S \\
b=a \pm 1}} \frac{a_{0}}{z_{a}-z_{S}}+\sum_{\substack{b \notin S \\
b \neq a}} \frac{k_{a} \cdot k_{b}}{z_{a}-z_{b}}-\sum_{\substack{b \notin S \\
b=a \pm 1}} \frac{a_{0}}{z_{a}-z_{b}}+\mathcal{O}(\epsilon), \quad a \notin S .
\end{aligned}
$$

Then, as in (2.16),

$$
\sum_{a \in S} \breve{g}_{a}(x, k)=\sum_{\substack{a, b \in S \\ b \neq a}} \frac{k_{a} \cdot k_{b}}{x_{a}-x_{b}}-\sum_{\substack{a, b \in S \\ b=a \pm 1}} \frac{a_{0}}{x_{a}-x_{b}}=0
$$

implying that if $\breve{g}_{a}=0$ for $a \in S, a \neq s$, where $s$ is some particular element of $S$, then it follows that $\breve{g}_{s}(x)=0$ as well.

Also, as in (2.17),

$$
\sum_{a \in S}\left(x_{a}-x_{r}\right) \breve{g}_{a}(x, k)=\sum_{\substack{a, b \in S \\ b \neq a}} \frac{x_{a}-x_{r}}{x_{a}-x_{b}} k_{a} \cdot k_{b}-\sum_{\substack{a, b \in S \\ b=a \pm 1}} \frac{x_{a}-x_{r}}{x_{a}-x_{b}} a_{0}=\frac{1}{2} k_{S}^{2}+n a_{0}, \quad k_{S}=\sum_{b \in S} k_{b},
$$

where $2 n$ is the number of $a$ for which $a+1 \notin S$ plus the number of $a$ for which $a-1 \notin S$. Then, if $\breve{g}_{a}(x, k)=0, a \in S, a \neq r, s$, as in (2.18), and noting that $m^{2}=-2 a_{0}$, we have that

$$
\left(x_{s}-x_{r}\right) \breve{g}_{s}(x, k)=\frac{1}{2}\left(k_{S}^{2}-n m^{2}\right)=\left(x_{r}-x_{s}\right) \breve{g}_{r}(x, k),
$$

and, if additionally $g_{r}(x, k)=0$, then $k_{S}^{2}=n m^{2}$. If $S$ is consecutive, and not the whole of $A$, then $n=1$, and this becomes the mass-shell condition.

Again, imposing the equations $\breve{f}_{a}(z, k)=0$, for all $a \in A$, and taking the limit $\epsilon \rightarrow 0$, we obtain, as in (2.19), a factorization into two sets of equations,

$$
\begin{aligned}
& \sum_{\substack{b \in S \\
b \neq a}} \frac{k_{a} \cdot k_{b}}{x_{a}-x_{b}}-\sum_{\substack{b \in S \\
b=a \pm 1}} \frac{a_{0}}{x_{a}-x_{b}}=0, \quad a \in S \\
& \frac{k_{S} \cdot k_{b}}{z_{a}-z_{S}}-\sum_{\substack{b \in S \\
b=a \pm 1}} \frac{a_{0}}{z_{a}-z_{S}}+\sum_{\substack{b \notin S \\
b \neq a}} \frac{k_{a} \cdot k_{b}}{z_{a}-z_{b}}-\sum_{\substack{b \notin S \\
b=a \pm 1}} \frac{a_{0}}{z_{a}-z_{b}}=0, \quad a \notin S,
\end{aligned}
$$

and, provided that $S$ is consecutive, the first set of equations are the modified CHY equations for the momenta $\left(k_{a}, a \in S ;-k_{S}\right)$, with associated variables $\left(x_{a}, a \in S ; \infty\right)$, and 
the second set are the equations for the momenta $\left(k_{S} ; k_{a}, a \notin S\right)$, with associated variables $\left(z_{S} ; z_{a}, a \notin S\right)$.

The results (B.2) to (B.8) make it straightforward to adapt the analysis of sections 3, 4 and 5 to the massive case. If we replace $\hat{f}_{a}(z, k)$ in $(3.1)$ by $\breve{f}_{a}(z, k)$ to obtain

$$
\mathcal{A}_{N}^{\phi, m}=\frac{1}{(-2)^{N-3}} \oint_{\mathcal{O}} \prod_{a \in A}^{\prime} \frac{1}{\breve{f}_{a}(z, k)} \prod_{a \in A} \frac{d z_{a}}{\left(z_{a}-z_{a+1}\right)^{2}} / d \omega
$$

the effect in general is to replace $k_{a} \cdot k_{b}$ by $k_{a} \cdot k_{b}-a_{0}$ whenever $b=a \pm 1$, so that, for example, (3.3) becomes

$$
\begin{aligned}
\mathcal{A}_{4}^{\phi, m} & =\frac{1}{2} \oint_{\mathcal{O}} \frac{d z}{(1-z) z\left[\left(k_{3} \cdot k_{2}-a_{0}\right) z+\left(k_{3} \cdot k_{4}-a_{0}\right)(z-1)\right]}, \\
& =\frac{k_{3} \cdot\left(k_{2}+k_{4}\right)-2 a_{0}}{2\left(k_{3} \cdot k_{2}-a_{0}\right)\left(k_{3} \cdot k_{4}-a_{0}\right)}=\frac{1}{s+m^{2}}+\frac{1}{t+m^{2}} .
\end{aligned}
$$

Further the effect is to replace $s_{a b}=\left(k_{a}+k_{b}\right)^{2}$ by $s_{a b}-m^{2}=\left(k_{a}+k_{b}\right)^{2}-m^{2}$ and $s_{a b c}=\left(k_{a}+k_{b}+k_{c}\right)^{2}$ by $s_{a b c}-m^{2}=\left(k_{a}+k_{b}+k_{c}\right)^{2}-m^{2}$ in (3.9) to (3.14) [Note that $a, b$ and $a, b, c$ are consecutive where they occur in $s_{a b}$ and $\left.s_{a b c}.\right]$, giving the correct result for $\mathcal{A}_{5}^{\phi, m}$. Because of (B.5) and (B.6), the effect is to replace $s_{m}$ with $s_{m}-m^{2}$ and $\bar{s}_{m}$ with $\bar{s}_{m}-m^{2}$ throughout sections 4 and 5 , so that those sections provide inductive proofs of that $\mathcal{A}_{N}^{\phi, m}$ provides the correct $N$-point tree amplitudes for massive $\phi^{3}$ theory.

Open Access. This article is distributed under the terms of the Creative Commons Attribution License (CC-BY 4.0), which permits any use, distribution and reproduction in any medium, provided the original author(s) and source are credited.

\section{References}

[1] F. Cachazo, S. He and E.Y. Yuan, Scattering of massless particles in arbitrary dimension, arXiv: 1307.2199 [INSPIRE].

[2] L. Dolan and P. Goddard, Complete equivalence between gluon tree amplitudes in twistor string theory and in gauge theory, JHEP 06 (2012) 030 [arXiv:1111.0950] [INSPIRE].

[3] N. Berkovits and E. Witten, Conformal supergravity in twistor-string theory, JHEP 08 (2004) 009 [hep-th/0406051] [INSPIRE].

[4] R. Britto, F. Cachazo, B. Feng and E. Witten, Direct proof of tree-level recursion relation in Yang-Mills theory, Phys. Rev. Lett. 94 (2005) 181602 [hep-th/0501052] [InSPIRE].

[5] F. Cachazo, S. He and E.Y. Yuan, Scattering of massless particles: scalars, gluons and gravitons, arXiv: 1309.0885 [INSPIRE].

[6] J. Scherk, Zero-slope limit of the dual resonance model, Nucl. Phys. B 31 (1971) 222 [INSPIRE].

[7] N. Arkani-Hamed and J. Kaplan, On tree amplitudes in gauge theory and gravity, JHEP 04 (2008) 076 [arXiv:0801.2385] [INSPIRE].

[8] L. Mason and D. Skinner, Ambitwistor strings and the scattering equations, arXiv:1311.2564 [INSPIRE].

[9] N. Berkovits, Infinite tension limit of the pure spinor superstring, JHEP 03 (2014) 017 [arXiv: 1311.4156] [INSPIRE]. 